\title{
A Review of the Botany, Phytochemistry, Pharmacology and Toxicology of Rubiae Radix et Rhizoma
}

\author{
Mingqiu Shan *, Sheng Yu, Hui Yan, Peidong Chen, Li Zhang and Anwei Ding * \\ Jiangsu Collaborative Innovation Center of Chinese Medicinal Resources Industrialization, National and Local \\ Collaborative Engineering Center of Chinese Medicinal Resources Industrialization and Formulae Innovative \\ Medicine, Nanjing University of Chinese Medicine, Nanjing 210023, China; yusheng1219@163.com (S.Y.); \\ glory-yan@163.com (H.Y.); chenpeidong1970@163.com (P.C.); zhangliguanxiong@163.com (L.Z.) \\ * Correspondences: shanmingqiu@163.com (M.S.); awding105@163.com (A.D.); \\ Tel.: +86-25-8581-1519 (M.S.); Fax: +86-25-8581-1524 (M.S.); Tel./Fax: +86-25-8581-1523 (A.D.)
}

Academic Editor: Thomas Efferth

Received: 22 October 2016; Accepted: 15 December 2016; Published: 20 December 2016

\begin{abstract}
Rubia cordifolia Linn (Rubiaceae) is a climbing perennial herbal plant, which is widely distributed in China and India. Its root and rhizome, Rubiae Radix et Rhizoma (called Qiancao in China and Indian madder in India), is a well known phytomedicine used for hematemesis, epistaxis, flooding, spotting, traumatic bleeding, amenorrhea caused by obstruction, joint impediment pain, swelling and pain caused by injuries from falls. In addition, it is a kind of pigment utilized as a food additive and a dye for wool or fiber. This review mainly concentrates on studies of the botany, phytochemistry, pharmacology and toxicology of this Traditional Chinese Medicine. The phytochemical evidences indicated that over a hundred chemical components have been found and isolated from the medicine, such as anthraquinones, naphthoquinones, triterpenoids, cyclic hexapeptides and others. These components are considered responsible for the various bioactivities of the herbal drug, including anti-oxidation, anti-inflammation, immunomodulation, antitumor, effects on coagulation-fibrinolysis system, neuroprotection and other effects. Additionally, based on these existing results, we also propose some interesting future research directions. Consequently, this review should help us to more comprehensively understand and to more fully utilize the herbal medicine Rubiae Radix et Rhizoma.
\end{abstract}

Keywords: botany; pharmacology; phytochemistry; review; Rubiae Radix et Rhizoma; toxicology

\section{Introduction}

Rubia cordifolia Linn (R. cordifolia, Rubiaceae) is a climbing perennial herbal plant widely distributed in China and India. As a medicinal plant, it was firstly recorded in a formula with endoconch of Sepiella maindroni in "Huangdi Neijing", the most important ancient book in Chinese Traditional Medicine (TCM). Rubiae Radix et Rhizoma (RRR), the dried root and rhizome of $R$. cordifolia, is a famous TCM used for thousands of years. The medicinal part of $R$. cordifolia was specificly presented for the first time in "Shennong Bencaojing", another classic work on plants and their uses. Since then, this phytomedicine has been employed for hematemesis, epistaxis, flooding, spotting, traumatic bleeding, amenorrhea caused by obstruction, joint impediment pain, swelling and pain caused by injuries from falls [1]. With its excellent activity to cool blood, eliminate stasis, stop bleeding and unblock meridians, it has been considered in "Bencao Gangmu" and other ancient medical books as an important herbal drug for curing the syndromes caused by blood heat. Thus, RRR is still listed in the Chinese Pharmacopeia [1]. During the past decades, more than one hundred chemical compounds such as anthraquinones, naphthoquinones, cyclic hexapeptides, terpenoids, polysaccharides and other 
compounds have been found in RRR, isolated and identified [2-10]. The range of its pharmacological activity also has been widened, covering antioxidation, neuroprotection, anti-inflammation, antitumor, and immunomodulation effects, etc [3,11-17].

Additionally, R. cordifolia is a well-known source of red plant dye for clothes and food items with a long history of use in China and India [18-22]. The earliest record of this was in "Shijing", an ancient book about over 2500 years ago. In China, RRR has been listed by the Ministry of Health as one of the substances that can be used in dietary supplements.

With the developments of science and technology and the introduction of many advanced experimental methods and instruments during the recent decades, a large number of studies have been performed on many aspects of RRR and a lot of achievements have been made, which were reported in many literatures. So, to summarize these studies and findings, we aim with this paper to provide a comprehensive and up-to-date review of Rubiae Radix et Rhizoma that covers the studies of its botany, phytochemistry, pharmacology and toxicology.

\section{Botany}

According to the Chinese Pharmacopeia [1], R. cordifolia Linn is the sole and authentic plant source of Rubiae Radix et Rhizoma. At present, R. cordifolia L. varstenophylla Franch, R. cordifolia L. varherbacea Chun et How, R. cordifolia L. varpratensis Maxim and R. cordifolia L. varmollis Chun et How are considered as the indigenous varieties of $R$. cordifolia Linn [23]. In China, it is widely distributed in most regions, including the provinces of Shaanxi, Henan, Anhui, Hebei, Shandong, Hubei, Jiangsu, Zhejiang, and so on. Among them, Weinan Shannxi and Songxian Henan are the most fundamental production areas, famous for their high production volume and quality [24]. In some areas of China, R. schumanniana pritz., R. yunnanensis Diels, R. membranacea Diels, R. tinctorum L. and so on are used as folk medicines rather than $R$. cordifolia Linn. Nevertheless, neither of them is the authentic one [25].

$R$. cordifolia is a climbing perennial herbal plant. The roots, which cluster in the soil, are aubergine or orange-red. The elongating and rough stems slightly lignify at the base. The branches are four-edge shaped and there are some anatropousspinules on the edges. The papery leaves are ovate or ovate-lanceolate and 2-6 cm long, 1-3 cm wide [24]. Sometimes there are sparse bristles on the rough-surfaced leaves. The cymes are in the axils or on the tops and are in the shapes of large and loose cones. The subsphaeroidal fruits with smooth surfaces, about $5 \mathrm{~cm}$ in diameter, are black or black-purple when ripening. Growing at the altitude of 570-1800 m, R. cordifolia is found along the roadside or riverside, on hillsides and in valleys [23]. Generally, the root and rhizome are collected in spring or autumn in the third or fourth year after cultivation [25]. Figure 1 shows R. cordifolia and Rubiae Radix et Rhizoma.

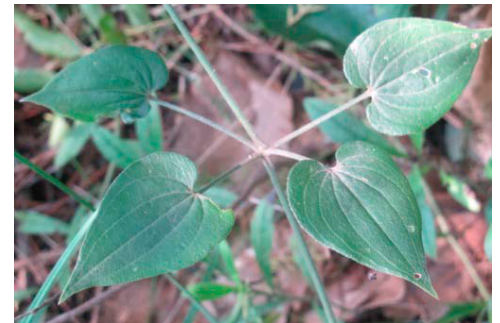

(A)

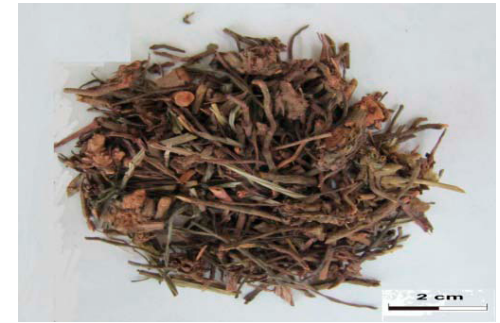

(B)

Figure 1. Rubia cordifolia Linn (A) and Rubiae Radix et Rhizoma (B).

\section{Phytochemistry}

More than a hundred components, which belong to anthraquinones, naphthoquinones, cyclic hexapeptides, terpenoids and other classes of compounds, have been found and isolated from RRR. Some of them have been proved responsible for the pharmacological activities in many studies. The compounds reported in the literature are listed in Table 1. 
Table 1. Compounds in Rubiae Radix et Rhizoma.

\begin{tabular}{|c|c|c|c|c|}
\hline Category & No. & Compound & $\begin{array}{l}\text { Molecular } \\
\text { Formula }\end{array}$ & Reference \\
\hline & 1 & Alizarin & $\mathrm{C}_{14} \mathrm{H}_{8} \mathrm{O}_{4}$ & {$[6,9,26-28]$} \\
\hline & 2 & Alizarin 2-methyl ether & $\mathrm{C}_{15} \mathrm{H}_{10} \mathrm{O}_{4}$ & [29] \\
\hline & 3 & Lucidinprimeveroside & $\mathrm{C}_{26} \mathrm{H}_{28} \mathrm{O}_{14}$ & {$[6,9,30]$} \\
\hline & 4 & Munjistin & $\mathrm{C}_{15} \mathrm{H}_{8} \mathrm{O}_{6}$ & {$[7,31]$} \\
\hline & 5 & Nordamnacanthal & $\mathrm{C}_{15} \mathrm{H}_{8} \mathrm{O}_{5}$ & [32] \\
\hline & 6 & Pupurin & $\mathrm{C}_{14} \mathrm{H}_{8} \mathrm{O}_{5}$ & {$[9,28,31]$} \\
\hline & 7 & Physcion & $\mathrm{C}_{16} \mathrm{H}_{12} \mathrm{O}_{5}$ & {$[32,33]$} \\
\hline & 8 & Ruberythric acid & $\mathrm{C}_{25} \mathrm{H}_{26} \mathrm{O}_{13}$ & {$[6,9,28,30]$} \\
\hline & 9 & Rubiacordone A & $\mathrm{C}_{23} \mathrm{H}_{22} \mathrm{O}_{10}$ & [34] \\
\hline & 10 & Rubiadin & $\mathrm{C}_{15} \mathrm{H}_{10} \mathrm{O}_{4}$ & {$[7,9,35]$} \\
\hline & 11 & Soranjidiol & $\mathrm{C}_{15} \mathrm{H}_{10} \mathrm{O}_{4}$ & [29] \\
\hline & 12 & Tectoquinone & $\mathrm{C}_{15} \mathrm{H}_{10} \mathrm{O}_{2}$ & {$[9,36-38]$} \\
\hline & 13 & Xanthopurpurin & $\mathrm{C}_{14} \mathrm{H}_{8} \mathrm{O}_{4}$ & {$[7,9,28,36]$} \\
\hline & 14 & 1-Hydroxyanthraquinone & $\mathrm{C}_{14} \mathrm{H}_{8} \mathrm{O}_{3}$ & [30] \\
\hline & 15 & 1-Hydroxy-2-methylanthraquinone & $\mathrm{C}_{15} \mathrm{H}_{10} \mathrm{O}_{3}$ & {$[6,7,9,32,36,37]$} \\
\hline & 16 & 1,4-Dihydroxy-6-methylanthraquinone & $\mathrm{C}_{15} \mathrm{H}_{10} \mathrm{O}_{4}$ & [32] \\
\hline & 17 & 1-Hydroxy-2-methoxyanthraquinone & $\mathrm{C}_{15} \mathrm{H}_{10} \mathrm{O}_{4}$ & [35] \\
\hline & 18 & 1,3-Dimethoxy-2-carboxylanthraquinone & $\mathrm{C}_{17} \mathrm{H}_{12} \mathrm{O}_{6}$ & [35] \\
\hline & 19 & 1,3,6-Trihydroxy-2-methylanthraquinone & $\mathrm{C}_{15} \mathrm{H}_{10} \mathrm{O}_{5}$ & {$[6-8,26,30,39]$} \\
\hline \multirow[t]{23}{*}{ Anthraquinones } & 20 & 1,4-dihydroxy-2-methylanthraquinone & $\mathrm{C}_{15} \mathrm{H}_{10} \mathrm{O}_{4}$ & {$[9,36,40]$} \\
\hline & 21 & 1,4-Dihydroxy-2,3-dimethylanthraquinone & $\mathrm{C}_{16} \mathrm{H}_{12} \mathrm{O}_{4}$ & [41] \\
\hline & 22 & 1,5-Dihydroxy-2-methylanthraquinone & $\mathrm{C}_{15} \mathrm{H}_{10} \mathrm{O}_{4}$ & [40] \\
\hline & 23 & 1,3-Dihydroxy-2-ethoxymethylanthraquinone & $\mathrm{C}_{17} \mathrm{H}_{14} \mathrm{O}_{5}$ & {$[6,41]$} \\
\hline & 24 & 1,4-Dihydroxy-2-methy-5-methoxylanthraquinone & $\mathrm{C}_{16} \mathrm{H}_{12} \mathrm{O}_{5}$ & [9] \\
\hline & 25 & 1-Acetoxy-3-methoxyanthraquinone & $\mathrm{C}_{17} \mathrm{H}_{12} \mathrm{O}_{5}$ & [29] \\
\hline & 26 & 1-Hydroxy-3-carbomethoxyanthraquinone & $\mathrm{C}_{16} \mathrm{H}_{10} \mathrm{O}_{5}$ & [36] \\
\hline & 27 & 1-Hydroxy-2-hydroxymethylanthraquinone & $\mathrm{C}_{15} \mathrm{H}_{10} \mathrm{O}_{4}$ & {$[8,36]$} \\
\hline & 28 & 1-Hydroxy-3-hydroxymethylanthraquinone & $\mathrm{C}_{15} \mathrm{H}_{10} \mathrm{O}_{4}$ & [41] \\
\hline & 29 & 1-Hydroxy-3-ethylanthraquinone & $\mathrm{C}_{16} \mathrm{H}_{12} \mathrm{O}_{3}$ & {$[41,42]$} \\
\hline & 30 & 1-Hydroxy-2,7-dimethylanthraquinone & $\mathrm{C}_{16} \mathrm{H}_{12} \mathrm{O}_{3}$ & [2] \\
\hline & 31 & 1,2,4,6-Tetrahydroxyanthraquinone & $\mathrm{C}_{14} \mathrm{H}_{8} \mathrm{O}_{6}$ & [43] \\
\hline & 32 & 1,2,4-Trihydroxylanthraquinone & $\mathrm{C}_{14} \mathrm{H}_{8} \mathrm{O}_{5}$ & [30] \\
\hline & 33 & 2-Hydroxy-6-methylanthraquinone & $\mathrm{C}_{15} \mathrm{H}_{10} \mathrm{O}_{3}$ & [2] \\
\hline & 34 & 2,6-Dihydroxyanthraquinone & $\mathrm{C}_{14} \mathrm{H}_{8} \mathrm{O}_{4}$ & [2] \\
\hline & 35 & 1,3,6-Trihydroxy-2-methyl-9,10-anthraquinone-3-O- $\beta$-glucoside & $\mathrm{C}_{21} \mathrm{H}_{20} \mathrm{O}_{10}$ & {$[7,30]$} \\
\hline & 36 & 1,3,6-Trihydroxy-2-methylanthraquinone-3-O- $\alpha$-rhamnosyl- $(1 \rightarrow 2)-\beta$-glucoside & $\mathrm{C}_{27} \mathrm{H}_{30} \mathrm{O}_{14}$ & {$[6,7,39]$} \\
\hline & 37 & 1,3,6-Trihydroxy-2-methylanthraquinone-3-O-(3'-O-acetyl)- $\alpha$-rhamnosyl- $(1 \rightarrow 2)-\beta$-glucoside & $\mathrm{C}_{29} \mathrm{H}_{32} \mathrm{O}_{15}$ & [7] \\
\hline & 38 & 1,3,6-Trihydroxy-2-methylanthraquinone-3-O-(6'-O-acetyl)- $\alpha$-rhamnosyl- $(1 \rightarrow 2)-\beta$-glucoside & $\mathrm{C}_{29} \mathrm{H}_{32} \mathrm{O}_{15}$ & {$[6,7]$} \\
\hline & 39 & $1,3,6$-Trihydroxy-2-methylanthraquinone-3- $O-\left(4^{\prime}, 6^{\prime}-O\right.$-diacetyl)- $\alpha$-rhamnosyl- $(1 \rightarrow 2)-\beta$-glucoside & $\mathrm{C}_{31} \mathrm{H}_{34} \mathrm{O}_{16}$ & [7] \\
\hline & 40 & 1,3,6-Trihydroxy-2-methylanthraquinone-3-O- $\left(3^{\prime}, 6^{\prime}-O\right.$-diacetyl $)-\alpha$-rhamnosyl- $(1 \rightarrow 2)-\beta$-glucoside & $\mathrm{C}_{31} \mathrm{H}_{34} \mathrm{O}_{16}$ & {$[7,39]$} \\
\hline & 41 & $1,3,6$-Trihydroxy-2-methylanthraquinone-3- $O-\left(6^{\prime}-O\right.$-acetyl)- $\alpha$-xylopyranosyl- $(1 \rightarrow 2)-\beta$-glucoside & $\mathrm{C}_{28} \mathrm{H}_{30} \mathrm{O}_{15}$ & [30] \\
\hline & 42 & 1,8-Dihydroxy-11,20(15-pentylnaphthaquinonyl) phenanthrene & $\mathrm{C}_{26} \mathrm{H}_{20} \mathrm{O}_{4}$ & [42] \\
\hline
\end{tabular}


Table 1. Cont

\begin{tabular}{|c|c|c|c|c|}
\hline Category & No. & Compound & $\begin{array}{l}\text { Molecular } \\
\text { Formula }\end{array}$ & Reference \\
\hline \multirow{18}{*}{ Naphthoquinones } & 43 & Mollugin & $\mathrm{C}_{17} \mathrm{H}_{16} \mathrm{O}_{4}$ & {$[6,7,9,36,44,45]$} \\
\hline & 44 & Furomollugin & $\mathrm{C}_{14} \mathrm{H}_{10} \mathrm{O}_{4}$ & {$[29,44-46]$} \\
\hline & 45 & $2^{\prime}$-Hydroxymollugin & $\mathrm{C}_{17} \mathrm{H}_{16} \mathrm{O}_{5}$ & {$[8,38]$} \\
\hline & 46 & $2^{\prime}$-Methoxymollugin & $\mathrm{C}_{18} \mathrm{H}_{18} \mathrm{O}_{5}$ & [8] \\
\hline & 47 & $1^{\prime}, 2^{\prime}$-Dihydroxydihydromollugin & $\mathrm{C}_{17} \mathrm{H}_{18} \mathrm{O}_{6}$ & [8] \\
\hline & 48 & $1^{\prime}$-Methoxy-2'-hydroxydihydromollugin & $\mathrm{C}_{18} \mathrm{H}_{20} \mathrm{O}_{6}$ & [8] \\
\hline & 49 & Epoxymollugin & $\mathrm{C}_{17} \mathrm{H}_{16} \mathrm{O}_{5}$ & [29] \\
\hline & 50 & Dihydromollugin & $\mathrm{C}_{17} \mathrm{H}_{18} \mathrm{O}_{4}$ & {$[7,33]$} \\
\hline & 51 & 3-Prenyl-5-methoxynaphthoquinone & $\mathrm{C}_{16} \mathrm{H}_{16} \mathrm{O}_{3}$ & [40] \\
\hline & 52 & 3-Prenyl-8-methoxynaphthoquinone & $\mathrm{C}_{16} \mathrm{H}_{16} \mathrm{O}_{3}$ & [40] \\
\hline & 53 & 2-Carbamoyl-3-hydroxynaphthoquinone & $\mathrm{C}_{11} \mathrm{H}_{7} \mathrm{NO}_{4}$ & [36] \\
\hline & 54 & 2-Carbnmoyl-3-methoxynaphthoquinone & $\mathrm{C}_{12} \mathrm{H}_{9} \mathrm{NO}_{4}$ & [36] \\
\hline & 55 & Dehydro- $\alpha$-lapachone & $\mathrm{C}_{15} \mathrm{H}_{12} \mathrm{O}_{3}$ & [36] \\
\hline & 56 & 2-Carboxymethyl-3-prenyl-2,3-epoxynaphthoquinone & $\mathrm{C}_{17} \mathrm{H}_{16} \mathrm{O}_{5}$ & {$[8,29,33,47]$} \\
\hline & 57 & 2-Carbomethoxy-3-(3'-hydroxy)isopentyl-1,4-naphthohydroquinone 4-O- $\beta$-glucoside & $\mathrm{C}_{23} \mathrm{H}_{30} \mathrm{O}_{10}$ & [7] \\
\hline & 58 & 2-Carbomethoxy-3-prenyl-1,4-naphthohydroquinone 1,4-di-O- $\beta$-glucoside & $\mathrm{C}_{29} \mathrm{H}_{38} \mathrm{O}_{14}$ & [7] \\
\hline & 59 & $\begin{array}{l}\text { 5-Hydroxy-2-[7-hydroxy-4-(1-hydroxy-1-methylethyl)-2-methyl-6-oxo-2,3,3a,6-tetrahydro-4H-1,5-dioxabenzo- } \\
\text { [de]anthracen-2-yl]-naphtho[1,2-b]furan-4-carboxylic acid methyl ester }\end{array}$ & $\mathrm{C}_{33} \mathrm{H}_{28} \mathrm{O}_{9}$ & [8] \\
\hline & 60 & $\begin{array}{l}\text { 6-Hydroxy-2-(5-hydroxy-4-methoxycarbonyl-naphtho-[1,2-b]furan-2-yl)-2-methyl-3,4-dihydro-2H-benzo[h]- } \\
\text { chromene-5-carboxylic acid methyl ester }\end{array}$ & $\mathrm{C}_{30} \mathrm{H}_{24} \mathrm{O}_{8}$ & [8] \\
\hline \multirow{16}{*}{$\begin{array}{c}\text { Cyclic } \\
\text { hexapeptides }\end{array}$} & 61 & RA-I & $\mathrm{C}_{40} \mathrm{H}_{48} \mathrm{~N}_{6} \mathrm{O}_{10}$ & {$[3,48]$} \\
\hline & 62 & RA-II & $\mathrm{C}_{40} \mathrm{H}_{48} \mathrm{~N}_{6} \mathrm{O}_{9}$ & [48] \\
\hline & 63 & RA-III & $\mathrm{C}_{41} \mathrm{H}_{50} \mathrm{~N}_{6} \mathrm{O}_{10}$ & {$[3,48]$} \\
\hline & 64 & RA-IV & $\mathrm{C}_{41} \mathrm{H}_{50} \mathrm{~N}_{6} \mathrm{O}_{10}$ & [48] \\
\hline & 65 & RA-V & $\mathrm{C}_{40} \mathrm{H}_{48} \mathrm{~N}_{6} \mathrm{O}_{9}$ & {$[3,48]$} \\
\hline & 66 & RA-VI & $\mathrm{C}_{41} \mathrm{H}_{50} \mathrm{~N}_{6} \mathrm{O}_{10}$ & [49] \\
\hline & 67 & RA-VII & $\mathrm{C}_{41} \mathrm{H}_{50} \mathrm{~N}_{6} \mathrm{O}_{9}$ & {$[3,48]$} \\
\hline & 68 & RA-VIII & $\mathrm{C}_{41} \mathrm{H}_{50} \mathrm{~N}_{6} \mathrm{O}_{10}$ & [49] \\
\hline & 69 & RA-IX & $\mathrm{C}_{43} \mathrm{H}_{51} \mathrm{~N}_{6} \mathrm{O}_{10}$ & [50] \\
\hline & 70 & RA-X & $\mathrm{C}_{43} \mathrm{H}_{52} \mathrm{~N}_{6} \mathrm{O}_{11}$ & [50] \\
\hline & 71 & RA-XI & $\mathrm{C}_{42} \mathrm{H}_{50} \mathrm{~N}_{6} \mathrm{O}_{11}$ & [51] \\
\hline & 72 & RA-XII & $\mathrm{C}_{46} \mathrm{H}_{58} \mathrm{~N}_{6} \mathrm{O}_{14}$ & [51] \\
\hline & 73 & RA-XIII & $\mathrm{C}_{48} \mathrm{H}_{60} \mathrm{~N}_{6} \mathrm{O}_{16}$ & [51] \\
\hline & 74 & RA-XIV & $\mathrm{C}_{48} \mathrm{H}_{58} \mathrm{~N}_{6} \mathrm{O}_{15}$ & [51] \\
\hline & 75 & RA-XV & $\mathrm{C}_{48} \mathrm{H}_{60} \mathrm{~N}_{6} \mathrm{O}_{15}$ & [52] \\
\hline & 76 & RA-XVI & $\mathrm{C}_{48} \mathrm{H}_{60} \mathrm{~N}_{6} \mathrm{O}_{16}$ & [52] \\
\hline
\end{tabular}


Table 1. Cont.

\begin{tabular}{|c|c|c|c|c|}
\hline \multirow[t]{21}{*}{ Category } & No. & Compound & $\begin{array}{c}\text { Molecular } \\
\text { Formula }\end{array}$ & Reference \\
\hline & 77 & RA-XVII & $\mathrm{C}_{41} \mathrm{H}_{50} \mathrm{~N}_{6} \mathrm{O}_{9}$ & [53] \\
\hline & 78 & RA-XVIII & $\mathrm{C}_{41} \mathrm{H}_{50} \mathrm{~N}_{6} \mathrm{O}_{10}$ & [54] \\
\hline & 79 & RA-XIX & $\mathrm{C}_{44} \mathrm{H}_{57} \mathrm{~N}_{6} \mathrm{O}_{9}$ & [55] \\
\hline & 80 & RA-XX & $\mathrm{C}_{42} \mathrm{H}_{52} \mathrm{~N}_{6} \mathrm{O}_{9}$ & [55] \\
\hline & 81 & RA-XXI & $\mathrm{C}_{41} \mathrm{H}_{50} \mathrm{~N}_{6} \mathrm{O}_{9}$ & [55] \\
\hline & 82 & RA-XXII & $\mathrm{C}_{41} \mathrm{H}_{50} \mathrm{~N}_{6} \mathrm{O}_{10}$ & [55] \\
\hline & 83 & RA-XXIII & $\mathrm{C}_{43} \mathrm{H}_{53} \mathrm{~N}_{7} \mathrm{O}_{10}$ & [56] \\
\hline & 84 & RA-XXIV & $\mathrm{C}_{42} \mathrm{H}_{51} \mathrm{~N}_{7} \mathrm{O}_{10}$ & [56] \\
\hline & 85 & Rubicordin A & $\mathrm{C}_{46} \mathrm{H}_{60} \mathrm{~N}_{6} \mathrm{O}_{14}$ & [3] \\
\hline & 86 & Rubicordin B & $\mathrm{C}_{47} \mathrm{H}_{62} \mathrm{~N}_{6} \mathrm{O}_{14}$ & [3] \\
\hline & 87 & Rubicordin C & $\mathrm{C}_{42} \mathrm{H}_{54} \mathrm{~N}_{6} \mathrm{O}_{9}$ & [3] \\
\hline & 88 & Rubiyunnanin B & $\mathrm{C}_{46} \mathrm{H}_{58} \mathrm{~N}_{6} \mathrm{O}_{14}$ & [3] \\
\hline & 89 & RY-II & $\mathrm{C}_{47} \mathrm{H}_{60} \mathrm{~N}_{6} \mathrm{O}_{14}$ & [3] \\
\hline & 90 & neo-RA-V & $\mathrm{C}_{40} \mathrm{H}_{48} \mathrm{~N}_{6} \mathrm{O}_{9}$ & [5] \\
\hline & 91 & allo-RA-V & $\mathrm{C}_{40} \mathrm{H}_{48} \mathrm{~N}_{6} \mathrm{O}_{9}$ & [5] \\
\hline & 92 & O-seco-RA-V & $\mathrm{C}_{40} \mathrm{H}_{50} \mathrm{~N}_{6} \mathrm{O}_{9}$ & [5] \\
\hline & 93 & O-seco-RA-XXIV & $\mathrm{C}_{42} \mathrm{H}_{53} \mathrm{~N}_{7} \mathrm{O}_{10}$ & {$[4]$} \\
\hline & 94 & RAI-III & $\mathrm{C}_{41} \mathrm{H}_{50} \mathrm{~N}_{6} \mathrm{O}_{10}$ & [57] \\
\hline & 95 & RAI-VI & $\mathrm{C}_{41} \mathrm{H}_{50} \mathrm{~N}_{6} \mathrm{O}_{10}$ & [57] \\
\hline & 96 & RA-dimer A & $\mathrm{C}_{80} \mathrm{H}_{94} \mathrm{~N}_{12} \mathrm{O}_{18}$ & [58] \\
\hline \multirow{12}{*}{ Triterpenoids } & 97 & Oleanolic acid & $\mathrm{C}_{30} \mathrm{H}_{48} \mathrm{O}_{3}$ & {$[29,33]$} \\
\hline & 98 & Oleanolic aldehyde acetate & $\mathrm{C}_{32} \mathrm{H}_{50} \mathrm{O}_{3}$ & [46] \\
\hline & 99 & Rubiarbonol A & $\mathrm{C}_{30} \mathrm{H}_{50} \mathrm{O}_{4}$ & {$[43,59]$} \\
\hline & 100 & Rubiarbonol B & $\mathrm{C}_{30} \mathrm{H}_{50} \mathrm{O}_{3}$ & [43] \\
\hline & 101 & Rubiatriol & $\mathrm{C}_{30} \mathrm{H}_{50} \mathrm{O}_{3}$ & [26] \\
\hline & 102 & Rubicoumaric acid & $\mathrm{C}_{39} \mathrm{H}_{54} \mathrm{O}_{6}$ & [10] \\
\hline & 1037 & Rubifolic acid & $\mathrm{C}_{30} \mathrm{H}_{48} \mathrm{O}_{4}$ & [10] \\
\hline & 104 & Rubiprasin A & $\mathrm{C}_{32} \mathrm{H}_{52} \mathrm{O}_{5}$ & {$[60]$} \\
\hline & 105 & Rubiprasin B & $\mathrm{C}_{32} \mathrm{H}_{52} \mathrm{O}_{4}$ & {$[60]$} \\
\hline & 106 & Rubiprasin C & $\mathrm{C}_{32} \mathrm{H}_{50} \mathrm{O}_{5}$ & [60] \\
\hline & 107 & Ursolic acid & $\mathrm{C}_{30} \mathrm{H}_{48} \mathrm{O}_{3}$ & [41] \\
\hline & 108 & $3-\beta$-Friedelinol & $\mathrm{C}_{30} \mathrm{H}_{52} \mathrm{O}_{6}$ & [61] \\
\hline \multirow{8}{*}{$\begin{array}{c}\text { Other } \\
\text { compounds }\end{array}$} & 109 & $\beta$-Sitostenone & $\mathrm{C}_{29} \mathrm{H}_{48} \mathrm{O}$ & [43] \\
\hline & 110 & $\beta$-Sitosterol & $\mathrm{C}_{29} \mathrm{H}_{50} \mathrm{O}$ & {$[33,41]$} \\
\hline & 111 & 5-Methoxygeniposidic acid & $\mathrm{C}_{17} \mathrm{H}_{24} \mathrm{O}_{11}$ & [62] \\
\hline & 112 & 6-Methoxygeniposidic acid & $\mathrm{C}_{17} \mathrm{H}_{24} \mathrm{O}_{11}$ & [46] \\
\hline & 113 & 3,5-di-(p-hydroxybenzyl)phenol & $\mathrm{C}_{20} \mathrm{H}_{18} \mathrm{O}_{3}$ & [2] \\
\hline & 114 & $n$-Heptadecane & $\mathrm{C}_{17} \mathrm{H}_{36}$ & [2] \\
\hline & 115 & $n$-Nonadecane & $\mathrm{C}_{19} \mathrm{H}_{40}$ & [2] \\
\hline & 116 & $(+)$-Lariciresinol & $\mathrm{C}_{20} \mathrm{H}_{24} \mathrm{O}_{6}$ & [38] \\
\hline
\end{tabular}


Table 1. Cont

\begin{tabular}{|c|c|c|c|c|}
\hline Category & No. & Compound & $\begin{array}{l}\text { Molecular } \\
\text { Formula }\end{array}$ & Reference \\
\hline \multirow{18}{*}{$\begin{array}{l}\text { Other } \\
\text { compounds }\end{array}$} & 117 & 3,3'-bis(3,4-Dihydro-4-hydroxy-6-methoxy-2H-1-benzopyran) & $\mathrm{C}_{20} \mathrm{H}_{22} \mathrm{O}_{6}$ & [29] \\
\hline & 118 & 8-Hydroxy $n$-pentadecanyl decan-4-en-1-oate & $\mathrm{C}_{25} \mathrm{H}_{48} \mathrm{O}_{3}$ & [2] \\
\hline & 119 & n-Octacosanyl octa-1-oate & $\mathrm{C}_{36} \mathrm{H}_{72} \mathrm{O}_{2}$ & [2] \\
\hline & 120 & Rubilactone & $\mathrm{C}_{15} \mathrm{H}_{10} \mathrm{O}_{5}$ & {$[41,45,63]$} \\
\hline & 121 & Rubioncolin B & $\mathrm{C}_{31} \mathrm{H}_{24} \mathrm{O}_{10}$ & [8] \\
\hline & 122 & 2,3-Dihydro-2-(4-hydroxy-3-methoxyphenyl)-3-hydroxymethyl-5- $\omega$-hydroxypropyl-7-methoxybenzofuran & $\mathrm{C}_{20} \mathrm{H}_{24} \mathrm{O}_{6}$ & [43] \\
\hline & 123 & Palmitic acid & $\mathrm{C}_{16} \mathrm{H}_{32} \mathrm{O}_{2}$ & [41] \\
\hline & 124 & Tricosanoic acid & $\mathrm{C}_{23} \mathrm{H}_{46} \mathrm{O}_{2}$ & [41] \\
\hline & 125 & Rubiasin A & $\mathrm{C}_{15} \mathrm{H}_{16} \mathrm{O}_{2}$ & {$[38,64]$} \\
\hline & 126 & Rubiasin B & $\mathrm{C}_{15} \mathrm{H}_{16} \mathrm{O}_{2}$ & [64] \\
\hline & 127 & Rubiasin C & $\mathrm{C}_{15} \mathrm{H}_{16} \mathrm{O}_{2}$ & {$[64]$} \\
\hline & 128 & Atraric acid & $\mathrm{C}_{10} \mathrm{H}_{12} \mathrm{O}_{4}$ & [61] \\
\hline & 129 & Vanillic acid & $\mathrm{C}_{8} \mathrm{H}_{8} \mathrm{O}_{4}$ & [61] \\
\hline & 130 & D-3-O-Methoxy-chiroinositol & $\mathrm{C}_{7} \mathrm{H}_{14} \mathrm{O}_{6}$ & [61] \\
\hline & 131 & Polysaccharide RPS-1 & Not mentioned & [65] \\
\hline & 132 & Polysaccharide RPS-2 & Not mentioned & [65] \\
\hline & 133 & Polysaccharide RPS-3 & Not mentioned & [65] \\
\hline & 134 & Polysaccharide QA2 & Not mentioned & {$[66]$} \\
\hline
\end{tabular}




\subsection{Anthraquinones}

Anthraquinone is a well-known category of phytochemicals. Alizarin, munjistin, purpurin, rubiadin, tectoquinone and xanthopurpurin are the common ones. Among them, purpurin is one of the two chemical markers that serve to evaluate the quality of the herbal medicine in the Chinese Pharmacopeia [1]. In pharmacological studies, it has been proved to have anti-angiogenic [67], and anti-oxidant [68,69] functions. Besides, these anthraquinonesare often found combined with glucoside, xylosyl-( $1 \rightarrow 6)$-glucoside (primeverose) or rhamnosyl- $(1 \rightarrow 2)$-glucoside to form the corresponding anthraquinone glycosides. The structures are shown in Figure 2.<smiles>[R]c1cc([R6])c2c(c1)C(=O)c1c([R])c([R])c([R])c([R])c1C2=O</smiles>

$\begin{array}{lllllll}1 & \mathrm{R}_{1}=\mathrm{OH} & \mathrm{R}_{2}=\mathrm{OH} & \mathrm{R}_{3}=\mathrm{H} & \mathrm{R}_{4}=\mathrm{H} & \mathrm{R}_{5}=\mathrm{H} & \mathrm{R}_{6}=\mathrm{H} \\ 2 & \mathrm{R}_{1}=\mathrm{OH} & \mathrm{R}_{2}=\mathrm{OCH}_{3} & \mathrm{R}_{3}=\mathrm{H} & \mathrm{R}_{4}=\mathrm{H} & \mathrm{R}_{5}=\mathrm{H} & \mathrm{R}_{6}=\mathrm{H} \\ 4 & \mathrm{R}_{1}=\mathrm{OH} & \mathrm{R}_{2}=\mathrm{COOH} & \mathrm{R}_{3}=\mathrm{OH} & \mathrm{R}_{4}=\mathrm{H} & \mathrm{R}_{5}=\mathrm{H} & \mathrm{R}_{6}=\mathrm{H} \\ 5 & \mathrm{R}_{1}=\mathrm{H} & \mathrm{R}_{2}=\mathrm{CHO} & \mathrm{R}_{3}=\mathrm{OH} & \mathrm{R}_{4}=\mathrm{H} & \mathrm{R}_{5}=\mathrm{H} & \mathrm{R}_{6}=\mathrm{H} \\ 6 & \mathrm{R}_{1}=\mathrm{OH} & \mathrm{R}_{2}=\mathrm{OH} & \mathrm{R}_{3}=\mathrm{H} & \mathrm{R}_{4}=\mathrm{OH} & \mathrm{R}_{5}=\mathrm{H} & \mathrm{R}_{6}=\mathrm{H} \\ 7 & \mathrm{R}_{1}=\mathrm{OH} & \mathrm{R}_{2}=\mathrm{H} & \mathrm{R}_{3}=\mathrm{CH}_{3} & \mathrm{R}_{4}=\mathrm{H} & \mathrm{R}_{5}=\mathrm{OCH}_{3} & \mathrm{R}_{6}=\mathrm{OH} \\ 9 & \mathrm{R}_{1}=\mathrm{OH} & \mathrm{R}_{2}=\mathrm{CH}_{3} & \mathrm{R}_{3}=\mathrm{O}-\mathrm{rha} & \mathrm{R}_{4}=\mathrm{H} & \mathrm{R}_{5}=\mathrm{OCOCH}_{3} & \mathrm{R}_{6}=\mathrm{H} \\ 10 & \mathrm{R}_{1}=\mathrm{OH} & \mathrm{R}_{2}=\mathrm{CH}_{3} & \mathrm{R}_{3}=\mathrm{OH} & \mathrm{R}_{4}=\mathrm{H} & \mathrm{R}_{5}=\mathrm{H} & \mathrm{R}_{6}=\mathrm{H} \\ 11 & \mathrm{R}_{1}=\mathrm{OH} & \mathrm{R}_{2}=\mathrm{H} & \mathrm{R}_{3}=\mathrm{CH}_{3} & \mathrm{R}_{4}=\mathrm{H} & \mathrm{R}_{5}=\mathrm{OH} & \mathrm{R}_{6}=\mathrm{H} \\ 12 & \mathrm{R}_{1}=\mathrm{H} & \mathrm{R}_{2}=\mathrm{CH} & \mathrm{R}_{3}=\mathrm{H} & \mathrm{R}_{4}=\mathrm{H} & \mathrm{R}_{5}=\mathrm{H} & \mathrm{R}_{6}=\mathrm{H} \\ 13 & \mathrm{R}_{1}=\mathrm{OH} & \mathrm{R}_{2}=\mathrm{H} & \mathrm{R}_{3}=\mathrm{OH} & \mathrm{R}_{4}=\mathrm{H} & \mathrm{R}_{5}=\mathrm{H} & \mathrm{R}_{6}=\mathrm{H}\end{array}$<smiles>O=C1c2ccccc2C(=O)c2c1cc(O[C@@H]1O[C@H](CO[C@H]3OC[C@@H](O)[C@H](O)[C@H]3O)[C@@H](O)[C@H](O)[C@H]1O)c(CO)c2O</smiles><smiles>O=C1c2ccccc2C(=O)c2c1ccc(O[C@@H]1O[C@H](CO[C@H]3OC[C@@H](O)[C@H](O)[C@H]3O)[C@@H](O)[C@H](O)[C@H]1O)c2O</smiles>

8

Figure 2. Structures of anthraquinones in Rubiae Radix et Rhizoma.

\subsection{Naphthoquinones}

Naphthoquinone is another category of phytochemicals in RRR. Mollugin is the representative on, reported to have anti-cancer [70,71], anti-inflammatory [17,72,73], and neuroprotective activities [72]. With a content of $10^{-3} \mathrm{~g} / \mathrm{g}$ level or even $10^{-2} \mathrm{~g} / \mathrm{g}$ level in RRR, it is designated as the other chemical marker by the Chinese Pharmacopeia [1]. Meanwhile, some derivatives of mollugin were isolated, too. The structures are shown in Figure 3. 
<smiles>COC(=O)c1c2c(c3ccccc3c1O)OC(C)(C)C=C2</smiles>

43<smiles>COC(=O)c1c2c(c3ccccc3c1O)OC(C)(C)C(O)C2O</smiles>

47<smiles>COC(=O)c1c(O)c2ccccc2c2occc12</smiles>

44<smiles>COC(=O)c1c2c(c3ccccc3c1O)OC(C)(C)C(O)C2OC</smiles>

48<smiles>COC(=O)c1c2c(c3ccccc3c1O)OC(C)(C)C(O)=C2</smiles>

45<smiles>COC(=O)c1c2c(c3ccccc3c1O)OC(C)(C)C1OC21</smiles>

49<smiles>COC(=O)c1c2c(c3ccccc3c1O)OC(C)(C)C(OC)=C2</smiles>

46<smiles>COC(=O)c1c2c(c3ccccc3c1O)OC(C)(C)CC2</smiles>

50

Figure 3. Structures of naphthoquinones in Rubiae Radix et Rhizoma.

Besides the naphthoquinone monomers above, three naphthohydroquinone dimers were also isolated, which were chemically named as 6-hydroxy-2-(5-hydroxy-4-methoxycarbonylnaphtho-[1,2- $b$ ] furan-2-yl)-2-methyl-3,4-dihydro-2H-benzo[ $h$ ]-chromene-5-carboxylic acid methyl ester, rubioncolin B and 5-hydroxy-2-[7-hydroxy-4-(1-hydroxy-1-methylethyl)-2-methyl-6-oxo-2,3,3a,6-tetrahydro-4H1,5-dioxa-benzo-[de]anthracen-2-yl]-naphtho[1,2-b]furan-4-carboxylic acid methyl ester.

\subsection{Cyclic Hexapeptides}

Some Japanese researchers were the first to isolate four cyclic hexapeptides from $R$. cordifolia and $R$. akane while screening for anticancer compounds in phytomedicines. Up to now, twenty four cyclic hexapeptides have been found and isolated from RRR, which were named as RA I-XXIV. Each RA contains both 18-member ring and 14-member ring systems, which consist of some amino acids including $\mathrm{N}$-methyl-O-methyl-L-tyrosine, pyroglutamic acid, L-alanine, D-alanine, etc. Among these RAs, RA-V and RA-VII were the dominating two, found at levels of nearly $100 \mu \mathrm{g} / \mathrm{g}$, while the others represent less than $1 \mu \mathrm{g} / \mathrm{g}$ in RRR. Furthermore, in recent years, some analogues or precursors of RAs were also isolated from RRR, such as neo-RA-V, allo-RA-V, rubicordin A-C, O-seco-RA-V, $O$-seco-RA-XXIV, and so on. The structures are shown in Figure 4.

\subsection{Triterpenoids}

Among phytomedicines, oleanolic acid and ursolic acid are common triterpenoids. The two compounds also have been found and isolated in RRR. Rubiprasin A-C, rubiarbonol A and B, rubicoumaric acid and rubifolic acid and other triterpenoids were isolated, too.

\subsection{Other Compounds}

Other than anthraquinones, naphthoquinones, cyclic hexapeptides and triterpenoids, phytochemistry researchers have found and isolated many other chemical compounds from RRR, including some organic acids, polysaccharides, rubilactone, rubiasin A-C, $\beta$-sitostenone, $\beta$-sitosterol, 5-methoxygeniposidic acid, 6-methoxygeniposidic acid, and so on. 


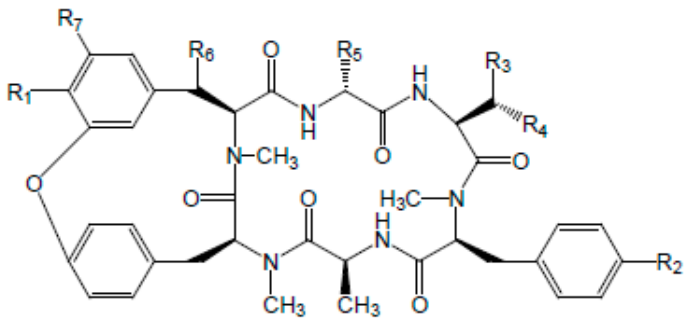

\begin{tabular}{|c|c|c|c|c|c|c|c|}
\hline 61 & $\mathrm{R}_{1}=\mathrm{OH}$ & $\mathrm{R}_{2}=\mathrm{OCH}_{3}$ & $\mathrm{R}_{3}=\mathrm{OH}$ & $\mathrm{R}_{4}=\mathrm{H}$ & $\mathrm{R}_{5}=\mathrm{CH}_{3}$ & $\mathrm{R}_{6}=\mathrm{H}$ & $\mathrm{R}_{7}=\mathrm{H}$ \\
\hline 62 & $\mathrm{R}_{1}=\mathrm{OCH}_{3}$ & $\mathrm{R}_{2}=\mathrm{OH}$ & $\mathrm{R}_{3}=\mathrm{H}$ & $\mathrm{R}_{4}=\mathrm{H}$ & $\mathrm{R}_{5}=\mathrm{CH}_{3}$ & $\mathrm{R}_{6}=\mathrm{H}$ & $\mathrm{R}_{7}=\mathrm{H}$ \\
\hline 63 & $\mathrm{R}_{1}=\mathrm{OCH}_{3}$ & $\mathrm{R}_{2}=\mathrm{OCH}_{3}$ & $\mathrm{R}_{3}=\mathrm{OH}$ & $\mathrm{R}_{4}=\mathrm{H}$ & $\mathrm{R}_{5}=\mathrm{CH}_{3}$ & $\mathrm{R}_{6}=\mathrm{H}$ & $\mathrm{R}_{7}=\mathrm{H}$ \\
\hline 64 & $\mathrm{R}_{1}=\mathrm{OCH}_{3}$ & $\mathrm{R}_{2}=\mathrm{OCH}_{3}$ & $\mathrm{R}_{3}=\mathrm{H}$ & $\mathrm{R}_{4}=\mathrm{H}$ & $\mathrm{R}_{5}=\mathrm{CH}_{3}$ & $\mathrm{R}_{6}=\mathrm{OH}$ & $\mathrm{R}_{7}=\mathrm{H}$ \\
\hline 65 & $\mathrm{R}_{1}=\mathrm{OH}$ & $\mathrm{R}_{2}=\mathrm{OCH}_{3}$ & $\mathrm{R}_{3}=\mathrm{H}$ & $R_{4}=H$ & $\mathrm{R}_{5}=\mathrm{CH}_{3}$ & $\mathrm{R}_{6}=\mathrm{H}$ & $\mathrm{R}_{7}=\mathrm{H}$ \\
\hline 67 & $\mathrm{R}_{1}=\mathrm{OCH}_{3}$ & $\mathrm{R}_{2}=\mathrm{OCH}_{3}$ & $\mathrm{R}_{3}=\mathrm{H}$ & $\mathrm{R}_{4}=\mathrm{H}$ & $\mathrm{R}_{5}=\mathrm{CH}_{3}$ & $\mathrm{R}_{6}=\mathrm{H}$ & $\mathrm{R}_{7}=\mathrm{H}$ \\
\hline 68 & $\mathrm{R}_{1}=\mathrm{OCH}_{3}$ & $\mathrm{R}_{2}=\mathrm{OCH}_{3}$ & $\mathrm{R}_{3}=\mathrm{CH}_{3}$ & $\mathrm{R}_{4}=\mathrm{OH}$ & $\mathrm{R}_{5}=\mathrm{CH}_{3}$ & $\mathrm{R}_{6}=\mathrm{H}$ & $\mathrm{R}_{7}=\mathrm{H}$ \\
\hline 70 & $\mathrm{R}_{1}=\mathrm{OCH}_{3}$ & $\mathrm{R}_{2}=\mathrm{OCH}_{3}$ & $\mathrm{R}_{3}=\mathrm{CH}_{2} \mathrm{COOH}$ & $\mathrm{R}_{4}=\mathrm{H}$ & $\mathrm{R}_{5}=\mathrm{CH}_{3}$ & $\mathrm{R}_{6}=\mathrm{H}$ & $\mathrm{R}_{7}=\mathrm{H}$ \\
\hline 71 & $\mathrm{R}_{1}=\mathrm{OH}$ & $\mathrm{R}_{2}=\mathrm{OCH}_{3}$ & $\mathrm{R}_{3}=\mathrm{CH}_{2} \mathrm{COOH}$ & $\mathrm{R}_{4}=\mathrm{H}$ & $\mathrm{R}_{5}=\mathrm{CH}_{3}$ & $\mathrm{R}_{6}=\mathrm{H}$ & $\mathrm{R}_{7}=\mathrm{H}$ \\
\hline 72 & $\mathrm{R}_{1}=\mathrm{OGlu}$ & $\mathrm{R}_{2}=\mathrm{OCH}_{3}$ & $\mathrm{R}_{3}=\mathrm{H}$ & $R_{4}=H$ & $\mathrm{R}_{5}=\mathrm{CH}_{3}$ & $\mathrm{R}_{6}=\mathrm{H}$ & $\mathrm{R}_{7}=\mathrm{H}$ \\
\hline 73 & $\mathrm{R}_{1}=\mathrm{OGlu}$ & $\mathrm{R}_{2}=\mathrm{OCH}_{3}$ & $\mathrm{R}_{3}=\mathrm{CH}_{2} \mathrm{COOH}$ & $\mathrm{R}_{4}=\mathrm{H}$ & $\mathrm{R}_{5}=\mathrm{CH}_{3}$ & $\mathrm{R}_{6}=\mathrm{H}$ & $\mathrm{R}_{7}=\mathrm{H}$ \\
\hline 75 & $\mathrm{R}_{1}=\mathrm{OGlu}(6-\mathrm{OAc})$ & $\mathrm{R}_{2}=\mathrm{OCH}_{3}$ & $\mathrm{R}_{3}=\mathrm{H}$ & $\mathrm{R}_{4}=\mathrm{H}$ & $\mathrm{R}_{5}=\mathrm{CH}_{3}$ & $\mathrm{R}_{6}=\mathrm{H}$ & $\mathrm{R}_{7}=\mathrm{H}$ \\
\hline 76 & $\mathrm{R}_{1}=\mathrm{OGlu}$ & $\mathrm{R}_{2}=\mathrm{OCH}_{3}$ & $\mathrm{R}_{3}=\mathrm{H}$ & $\mathrm{R}_{4}=\mathrm{H}$ & $\mathrm{R}_{5}=\mathrm{CH}_{3}$ & $\mathrm{R}_{6}=\mathrm{OCOCH}_{3}$ & $\mathrm{R}_{7}=\mathrm{H}$ \\
\hline 77 & $\mathrm{R}_{1}=\mathrm{OH}$ & $\mathrm{R}_{2}=\mathrm{OCH}_{3}$ & $\mathrm{R}_{3}=\mathrm{H}$ & $\mathrm{R}_{4}=\mathrm{H}$ & $\mathrm{R}_{5}=\mathrm{CH}_{2} \mathrm{CH}_{3}$ & $\mathrm{R}_{6}=\mathrm{H}$ & $\mathrm{R}_{7}=\mathrm{H}$ \\
\hline 78 & $\mathrm{R}_{1}=\mathrm{OCH}_{3}$ & $\mathrm{R}_{2}=\mathrm{OCH}_{3}$ & $\mathrm{R}_{3}=\mathrm{H}$ & $\mathrm{R}_{4}=\mathrm{H}$ & $\mathrm{R}_{5}=\mathrm{CH}_{3}$ & $\mathrm{R}_{6}=\mathrm{H}$ & $\mathrm{R}_{7}=\mathrm{OH}$ \\
\hline 79 & $\mathrm{R}_{1}=\mathrm{OCH}_{3}$ & $\mathrm{R}_{2}=\mathrm{OCH}_{3}$ & $\mathrm{R}_{3}=\mathrm{CH}_{2}\left(\mathrm{CH}_{3}\right)_{2}$ & $\mathrm{R}_{4}=\mathrm{H}$ & $\mathrm{R}_{5}=\mathrm{CH}_{3}$ & $\mathrm{R}_{6}=\mathrm{H}$ & $\mathrm{R}_{7}=\mathrm{H}$ \\
\hline 80 & $\mathrm{R}_{1}=\mathrm{OCH}_{3}$ & $\mathrm{R}_{2}=\mathrm{OCH}_{3}$ & $\mathrm{R}_{3}=\mathrm{CH}_{3}$ & $\mathrm{R}_{4}=\mathrm{H}$ & $\mathrm{R}_{5}=\mathrm{CH}_{3}$ & $\mathrm{R}_{6}=\mathrm{H}$ & $\mathrm{R}_{7}=\mathrm{H}$ \\
\hline 81 & $\mathrm{R}_{1}=\mathrm{OH}$ & $\mathrm{R}_{2}=\mathrm{OCH}_{3}$ & $\mathrm{R}_{3}=\mathrm{CH}_{3}$ & $\mathrm{R}_{4}=\mathrm{H}$ & $\mathrm{R}_{5}=\mathrm{CH}_{3}$ & $\mathrm{R}_{6}=\mathrm{H}$ & $\mathrm{R}_{7}=\mathrm{H}$ \\
\hline 82 & $\mathrm{R}_{1}=\mathrm{OH}$ & $\mathrm{R}_{2}=\mathrm{OCH}_{3}$ & $\mathrm{R}_{3}=\mathrm{CH}_{3}$ & $\mathrm{R}_{4}=\mathrm{OH}$ & $\mathrm{R}_{5}=\mathrm{CH}_{3}$ & $\mathrm{R}_{6}=\mathrm{H}$ & $\mathrm{R}_{7}=\mathrm{H}$ \\
\hline 83 & $\mathrm{R}_{1}=\mathrm{OCH}_{3}$ & $\mathrm{R}_{2}=\mathrm{OCH}_{3}$ & $\mathrm{R}_{3}=\mathrm{CH}_{2} \mathrm{CONH}_{2}$ & $\mathrm{R}_{4}=\mathrm{H}$ & $\mathrm{R}_{5}=\mathrm{CH}_{3}$ & $\mathrm{R}_{6}=\mathrm{H}$ & $\mathrm{R}_{7}=\mathrm{H}$ \\
\hline 84 & $\mathrm{R}_{1}=\mathrm{OH}$ & $\mathrm{R}_{2}=\mathrm{OCH}_{3}$ & $\mathrm{R}_{3}=\mathrm{CH}_{2} \mathrm{CONH}_{2}$ & $\mathrm{R}_{4}=\mathrm{H}$ & $\mathrm{R}_{5}=\mathrm{CH}_{3}$ & $\mathrm{R}_{6}=\mathrm{H}$ & $\mathrm{R}_{7}=\mathrm{H}$ \\
\hline 39 & $R_{1}=$ OGlu & $\mathrm{R}_{2}=\mathrm{OCH}_{3}$ & $\mathrm{R}_{3}=\mathrm{CH}_{3}$ & $R_{1}=H$ & $\mathrm{R}_{5}=\mathrm{CH}_{3}$ & $R_{6}=H$ & $R_{7}=H$ \\
\hline
\end{tabular}
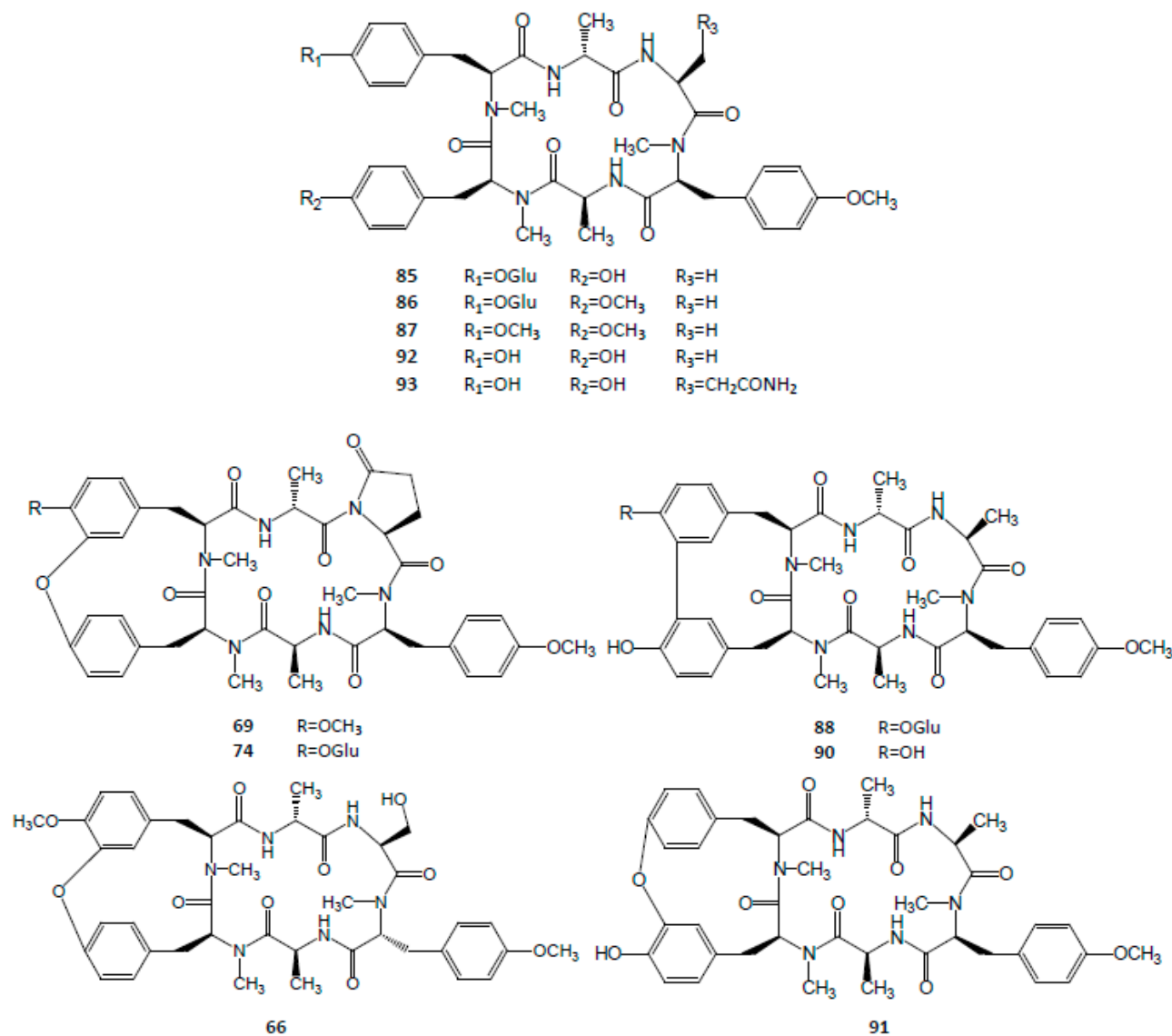

Figure 4. Structures of cyclic hexapeptides in Rubiae Radix et Rhizoma. 


\section{Pharmacology}

In many early pharmacological studies, RRR has been proved to have various functions, such as radioprotective [74], antidiabetic [75], hepatoprotective [76], antitumor [48,50-52,54,77], antiplatelet [78] effects, and so on [44], which were reported in previous reviews [79,80]. However, in recent years, some new activities and mechanisms were found that had not been previously reviewed.

\subsection{Effects on the Coagulation-Fibrinolysis System}

In TCM, RRR is well known for its activity of cooling blood, eliminating stasis, stopping bleeding and unblocking meridians [1]. In our previous study, we produced a rat model with blood stasis using a subcutaneous injection of adrenaline plus an ice water bath. After the model rats were treated with RRR, whole blood viscosity and plasma viscosity decreased and content of fibrinogen increased. In addition, shortened prothrombin time, prolonged thrombin time and activated partial thromboplastin time in the model rats were rectified after treatment with RRR. Furthermore, compared to model rats, RRR treatment also downregulated thromboxane $\mathrm{B}_{2}\left(\mathrm{TXB}_{2}\right)$ levels and upregulated 6-keto-PGF1 $\alpha$ levels [81]. Evidence of a study on the coagulation-fibrinolytic system of normal rats revealed that RRR decotion elevated the activity of tissue plasminogen activator (t-PA) [82].All these findings prove the effects of RRR on the blood system.

As we know, plasma hyaluronan-binding protein (PHBP) is a serine protease able to activate coagulation factor VII and prourokinase and to circulate as a single-chain form. With spermidine or heparin, it is autoproteolytically converted into an active two-chain form. In a screening investigation, purpurin was identified as a specific inhibitor of spermidine-induced autoactivation of PHBP [83].

\subsection{Antitumor}

RRR methanol extract (RRRME) was found to inhibit human laryngeal carcinoma cell (HEp-2 cell) proliferation and lactate dehydrogenase (LDH) release, to decrease reduced glutathione (GSH), glutathione S-transferase (GST) and protein levels, and to increase lipid peroxidation (LPO) in a dose-dependent manner. Further fluorescent microscopy and transmission electron microscopy confirmed this apoptotic effect [15].

Many studies have demonstrated that the cyclichexapeptides were the bioactive components responsible for the antitumor activity of RRR. The majority of the RA series compounds showed cytotoxicity against many cancer cells, including P-388 leukaemia cells [64], SGC-7901 human gastric adenocarcinoma cells, A-549 human non-small cell lung carcinoma cells, and Hela (human cervical carcinoma) cells [3].

Some small molecule compounds in RRR are also important antitumor compounds. As an Ames assay showed, alizarin was found effective in reducing his+ revertants induced by 4-nitro-o-phenylenediamine and 2-aminofluorene. Comet assay results indicated that DNA damage induced by $\mathrm{H}_{2} \mathrm{O}_{2}$ and 4-nitroquinoline-1-oxide could be reduced by anthraquinone too [84]. Mollugin was also proved to potentiate autophagic activity, induce growth inhibition and apoptosis of HN4 human oral cancer cells and SK-BR-3 breast cancer cells. It could upregulate the expression of mammalian target of rapamycin (mTOR), downregulate fatty acid synthase(FAS) gene expression and activate nuclear factor-E2-related factor 2 (Nrf2) with heme oxygenase-1 (HO-1)via some signaling pathways, such as phosphatidylinositol 3-kinase/protein kinase B (PI3K/AKT) and extracellular signal-regulated kinases (ERK). This naphthoquinone could also inhibit the activation of nuclear factor- $\mathrm{kB}(\mathrm{NF}-\mathrm{kB})$ and NF- $\mathrm{kB}$-dependent gene products involving antiapoptosis (Bcl-2 and Bcl-xl), invasion (MMP-9 and ICAM-1), and angiogenesis (FGF-2 and VEGF) [70,71,85]. 1-Hydroxy-2methylanthraquinone was another compound from RRR exhibiting cytotoxic effects on A375 malignant skin melanoma cells [86]. 


\subsection{Immunomodulation}

RRR ethanol extract (RRREE) was found to have protective effect against immunosuppression of Swiss albino mice induced by lead nitrate. This immunomodulation was considered associated to the increasing of macrophagocyte number and phagocyticindex, immunoglobulin levels and plaque-forming cell number [13]. In the serum of most peanut-allergy patients, there were increased levels of immunoglobulin E (IgE). After animals of a peanut-anaphylaxis mice model were treated with RRR aqueous extract, peanut-triggered anaphylactic reactions and plasma histamine levels decreased significantly, as did IgE production by a human B-cell line [14].

\subsection{Anti-Inflammation}

Pharmacological evidences showed that RRREE was able to ameliorate the lead nitrate-induced oxidative damage by improving the activities of superoxidedismutase (SOD) and catalase (CAT), increasing the content of GSH, and suppressing LPO [13]. In another study of indomethacin-induced enterocolitis in rats, some acute intestinal inflammation effects such as bowel wall thickening, mesenteric haemorrhage, mesentery adhesion and multiple mucosal ulcers of small intestine and colon emerged. Serum LDH activity increased, too. These disorders and changes were ameliorated after RRREE-treatment [87].

After RAW 264.7 macrophages were stimulated by LPS, the contents of NO, inducible nitric oxide synthase (iNOS), interleukin-1 $\beta$ (IL-1 $\beta$ ) and interleukin-6 (IL-6) increased significantly. However, mollugin-incubation brought some reduction of these inflammatory mediators [17]. In a screening investigation of some phytochemicals, physcion and 1-hydroxy-2methylanthraquinone showed amelioration on the damage to mouse peritoneal macrophages induced by lipo-polysaccharide (LPS) and interferon- $\gamma($ IFN- $\gamma)$, which was mediated through inhibition of iNOS protein expression and reduction of $\mathrm{NO}$ content [86].

\subsection{Neuroprotection}

In a study of reserpine-induced orofacial dyskinesia, RRRME-treatment was able to significantly inhibit vacuous chewing movements, tongue protrusions, orofacial bursts, catalepsy and to increase locomotion and rearing in an open field test. Meanwhile, bioassay results revealed that RRRME-treatment increased the levels of SOD, CAT, GSH, inhibited LPO and elevated dopamine levels in the forebrain region, compared with the model. These findings demonstrated that the neuroprotection of RRRME was related to its antioxidant activity [88].

Mollugin was considered as a neuroprotective agent for glutamate-induced neurotoxicityin the mousehippocampal HT22 cell line. The neuroprotection may be mediated by the effects on suppression of pro-inflammatory mediators, up-regulation of the expression ofHO-1 and the activity of $\mathrm{HO}$, nuclear accumulation of Nrf2 and activation of p38 mitogen-activated protein kinase (MAPK) pathway [72]. In the T-REx293human embryonic kidney cell line, A $\beta(42)$-EGFP (enhanced green fluorescent protein) plays a key role in Alzheimer's disease. Abnormal accumulation of A $\beta(42)$-EGFP would lead to apoptosis of this cell line. With treatment by RRR polysaccharides, A $\beta(42)$-EGFP accumulation decreased and cell activity was restored dramatically. Moreover, RRR polysaccharides inhibited cytotoxicity of $A \beta(42)-E G F P$, which may be mediated by potentiated degradation of proteosome [11].

\subsection{Antioxidation}

Results of studies on the gastroprotective effect on aspirin plus pylorus-ligated ulcer indicated that RRRME and its chloroform fraction brought notable decreases of ulcer index, total acidity, protein, pepsin content of the gastric fluid and increase of the mucin content. Among the key antioxidant parameters, after treatment with RRRME or its chloroform fraction, were a significant reduction in LPO and elevations in CAT, SOD, and GSH. It was concluded that the protective mechanism could perhaps be partly attributed to the effects of the herbal medicine on oxidative stress [12]. 
In $N$-nitrosodiethylamine-induced hepatocellular carcinoma rats, the activities of serum marker enzymes including aspartate transaminase (AST), alanine aminotransferase (ALT), alkaline phosphatase (ALP) and LDH with the levels of LPO and hydroxyl radicals in liver increased significantly. The opposite trends were seen with the activities of the antioxidants including SOD, CAT, GSH, GST, glutathione peroxidase (GPx) in liver and the levels of mitochondrial enzymes like isocitrate dehydrogenase (ICDH), succinate dehydrogenase (SDH), $\alpha$-ketoglutarate dehydrogenase ( $\alpha$-KGDH) and respiratory chain enzymes like nicotinamide adenine dinucleotide (NADH) dehydrogenase and cytochrome c oxidase. However, all these changes in the model group were markedly and dose-dependently ameliorated by treatment of RRRME, which indicated RRR perhaps could be used as an antioxidant for the treatment of some cancers [16]. Structure-radical scavenging activity relationship results demonstrated that hydroxyl groups on the benzene rings were essential to the radical scavenging function of hydroxylanthraquinone [89].

\subsection{Other Pharmacological Functions}

Besides the effects above, RRR have exhibited good effects in studies of anti-urolithiasis, anti-psoriasis, anti-nephrotoxicity, estrogenic and progestational activity, and so on [47,90-95] (Table 2).

\section{Toxicology}

Though some extracts or compounds from RRR have shown antitumor effects, rubiadin was reported to display carcinogenic potential. In the outer medulla, cytoplasmic swelling with basophilic changes and karyomegaly were observed in male F344 rats fed with rubiadin for one week whereas 26-week oral administration of the component induced atypical tubules, putative pre-neoplastic lesions, and karyomegaly. The results indicated that rubiadin may be a potent carcinogenic ingredient that targeted the proximal tubule cells in the outer medulla [96]. Rubiadin was also considered as both initiator and promoter of carcinogenicity targeting kidney, liver and large intestine [97]. In madder pigment, alizarin, purpurin and 1-hydroxyanthraquinone were found to have similar effects as ethidium bromide, a typical DNA intercalator. They exhibited potential genotoxicity by implanting into the DNA of Escherichia coli, blocking gene expression and inducing cell death [98].

\section{Conclusions and Remarks on Future Work}

As one of the earliest TCMs used in the clinic, RRR has shown various actions on many syndromes and indications for over 2000 years. In this paper, we have provided a review of RRR focused on the fields of botany, phytochemistry, pharmacology and toxicology based on the data and results collected from a large amount of research studies.

Rubidate is a synthetic derivative of ruberythric acid. It was documented to have the property of increasing leukocyte levels in peripheral blood $[99,100]$ and developed into a medicine for the treatment of the leucopenia [101] in China in the 1980s. At present, over 100 components have been isolated from RRR, including anthraquinones, naphthoquinones, cyclic hexapeptides and triterpenoids, etc. In structure-effect relationship studies, with bioactivity-guided and high-throughput screening methods, other components from RRR or their synthetic derivatives may potentially be found as candidate drugs like rubidate.

In traditional clinical use, RRR has a long history of use in some hemorrhages induced by blood stasis for its activities of stopping bleeding and resolving stasis, which had been proved by some pharmacological experiments [74-76]. However, according to modern pharmacology, the two effects are contradictory on some indicators of coagulative and fibrinolytic systems. In a pathological state, stanching would cause stasis and blood-activating would induce hemorrhages, so there still remain some questions to be answered, like what are the active components or fractions responsible for these two seemingly conflicting functions, respectively? How can they act to maintain a healthy state of relative equilibrium rather than pathological state of hemorrhage or stasis? 
Table 2. Other functions of Rubiae Radix et Rhizoma.

\begin{tabular}{|c|c|c|c|c|c|}
\hline Function & Inducer & Test Drug & Model & Efficacy Evaluation & Reference \\
\hline \multirow{3}{*}{ Anti-adipogenic activity } & & \multirow{3}{*}{$\begin{array}{l}\text { 2-Carboxymethyl-3-prenyl- } \\
\text { 2,3-epoxynaphthoquinone }\end{array}$} & \multirow{3}{*}{ 3T3-L1 preadipocytes } & Induced MMP loss, caspase- 3 activation & \multirow{3}{*}{ [54] } \\
\hline & & & & $\begin{array}{l}\text { Reduced differentiation-associated accumulation of } \\
\text { intracellular lipid }\end{array}$ & \\
\hline & & & & $\begin{array}{l}\text { Downregulatedexpressions of CCAAT/enhancer binding } \\
\text { protein- } \alpha \text {, PPAR } \gamma 1 \text {, PPAR } \gamma 2 \text {, adiponectin }\end{array}$ & \\
\hline Anti-urolithiasis & Ethylene glycol & Ethanol extract & Male Wistar albino rats & $\begin{array}{l}\text { Decreased calcium, oxalate levels and number of calcium } \\
\text { oxalate crystals deposits in kidney tissue }\end{array}$ & [90] \\
\hline \multirow{3}{*}{ Anti-psoriasis } & & \multirow{3}{*}{$\begin{array}{l}\text { Ethyl acetate fraction of } \\
\text { ethanol extract }\end{array}$} & \multirow{2}{*}{ HaCaT cells } & Decreased MMP & \multirow{3}{*}{ [91] } \\
\hline & & & & Induced apoptosis & \\
\hline & & & Male BALB/c mice & Increased NGL, TGL and VET & \\
\hline \multirow{3}{*}{ Anti-nephrotoxicity } & \multirow{3}{*}{ Cisplatin } & \multirow{3}{*}{ Ethanol extract } & \multirow{3}{*}{ Swiss albino mice } & Decreased values of serum urea and creatinine & \multirow{3}{*}{ [92] } \\
\hline & & & & Increased GPx, SOD and CAT & \\
\hline & & & & Inhibited LPO in kidney and liver & \\
\hline \multirow{2}{*}{$\begin{array}{c}\text { Estrogenic and } \\
\text { progestational activity }\end{array}$} & & \multirow{2}{*}{$\begin{array}{l}\text { Ethyl acetate precipitate } \\
\text { of methanol extract }\end{array}$} & \multirow{2}{*}{ Old female albino rats } & Increased the regularity of the estrous cycle & \multirow{2}{*}{ [93] } \\
\hline & & & & Increased uterine weight and foetal survival & \\
\hline \multirow{4}{*}{ Anti-osteoclastogenesis } & \multirow{4}{*}{ NF- $\kappa B$ ligand } & \multirow{4}{*}{ Mollugin } & \multirow{4}{*}{ Mice BMMs } & Inhibited osteoclast differentiation & \multirow{4}{*}{ [94] } \\
\hline & & & & Reduced the phosphorylation ofMAP kinase, Akt, and GSK3 $\beta$ & \\
\hline & & & & Inhibited expression of c-Fos, NFATc1, & \\
\hline & & & & $\begin{array}{l}\text { OSCAR, TRAP, DC-STAMP, OC-STAMP, integrin } \alpha v \text {, integrin } \\
\beta 3 \text {, cathepsin K, and ICAM- } 1\end{array}$ & \\
\hline Anti-HIV & HIV-1NL4.3 & Ethyl acetate extract & CEM-GFP cells & Reduced viral production & [95] \\
\hline
\end{tabular}


There is no doubt that RRR is an effective TCM in clinical practice with a long history and plenty of accumulated experiences. Nevertheless, the mutagenicity or carcinogenic potential of the active anthraquinones is a matter of concern [102,103]. At present, there is lack of research and literature on the in vivo metabolism and the metabolites of these components, so in terms of safe medication, the Absorption-Distribution-Metabolism-Excretion-Toxicity (ADMET) of these naturally occurring anthraquinones should be the urgent concern right now.

The present literature review provides a full-scale profile of various aspects of Rubiae Radix et Rhizoma and proposes some issues worth investigating in the future. We believe that it will help us to comprehensively understand and more effectively develop this traditional phytomedicine.

Acknowledgments: We are thankful for financially support from the National Natural Science Foundation of China (81473348), Priority Academic Program Development of Jiangsu Higher Education Institutions (PAPD-2014), Top-notch Academic Programs Project of Jiangsu Higher Education Institutions (TAPP-PPZY2015A070) and Key Research Project of Jiangsu Collaborative Innovation Center of Chinese Medicinal Resources Industrialization (ZDXM-1-3).

Author Contributions: Mingqiu Shan and Anwei Ding designed the paper; Mingqiu Shan, Peidong Chen and Hui Yan collected literatures on botany and phytochemistry; Li Zhang and Sheng Yu collected literatures on pharmacology and toxicology; Mingqiu Shan wrote the paper.

Conflicts of Interest: The authors declare no conflict of interest.

\section{References}

1. Chinese Pharmacopeia Commission. Pharmacopoeia of the People's Republic of China; Chinese Medical Science Press: Beijing, China, 2015; Volume 1, pp. 234-235.

2. Akhtar, M.S.; Ali, M.; Madhurima; Mir, S.R.; Singh, O. New anthraquinones from Rubia cordifolia roots. Indian J. Chem. B 2006, 45, 1945-1950. [CrossRef]

3. Chen, X.Q.; Zhao, S.M.; Wang, Z.; Zeng, G.Z.; Huang, M.B.; Tan, N.H. Rubicordins A-C, new cyclopeptides from Rubia cordifolia with cytotoxicity and inhibiting NF-kB signaling pathway. Tetrahedron 2015, 71, 9673-9678. [CrossRef]

4. Hitotsuyanagi, Y.; Kusano, J.; Kim, I.H.; Hasuda, T.; Fukaya, H.; Takeya, K. O-Seco-RA-XXIV, a possible precursor of an antitumor peptide RA-XXIV, from Rubia cordifolia L. Phytochem. Lett. 2012, 5, 335-339. [CrossRef]

5. Hitotsuyanagi, Y.; Odagiri, M.; Kato, S.; Kusano, J.; Hasuda, T.; Fukaya, H.; Takeya, K. Isolation, structure determination, and synthesis of allo-RA-V and neo-RA-V, RA-series bicyclic peptides from Rubia cordifolia $\mathrm{L}$. Chem. Eur. J. 2012, 18, 2839-2846. [CrossRef] [PubMed]

6. Itokawa, H.; Mihara, K.; Takeya, K. Studies on a novel anthraquinone and its glycosides isolated from Rubia cordifolia and R. akane. Chem. Pharm. Bull. 1983, 31, 2353-2358. [CrossRef]

7. Itokawa, H.; Qiao, Y.F.; Takeya, K. Anthraquinones and naphthohydroquinones from Rubia cordifolia. Phytochemistry 1989, 28, 3465-3468. [CrossRef]

8. Itokawa, H.; Ibraheim, Z.Z.; Qiao, Y.F.; Takeya, K. Anthraquinones, naphthohydroquinones and naphthohydroquinone dimers from Rubia cordifolia and their cytotoxic activity. Chem. Pharm. Bull. 1993, 41, 1869-1872. [CrossRef] [PubMed]

9. Singh, R. Isolation and synthesis of anthraquinones and related compounds of Rubia cordifolia. J. Serbian Chem. Soc. 2005, 70, 937-942. [CrossRef]

10. Talapatra, S.K.; Sarkar, A.C.; Talapatra, B. Two pentacyclic triterpenes from Rubia cordifolia. Phytochemistry 1981, 20, 1923-1927. [CrossRef]

11. Chakrabortee, S.; Liu, Y.; Zhang, L.; Matthews, H.R.; Zhang, H.R.; Pan, N.; Cheng, C.R.; Guan, S.H.; Guo, D.A.; Huang, Z.B. Macromolecular and small-molecule modulation of intracellular A beta(42)aggregationand associated toxicity. Biochem. J. 2012, 442, 507-515. [CrossRef] [PubMed]

12. Deoda, R.S.; Kumar, D.; Bhujbal, S.S. Gastroprotective effect of Rubia cordifolia Linn on aspirin plus pylorus-ligated ulcer. Evid. Based Complement. Altern. Med. 2011. [CrossRef] [PubMed]

13. Lodi, S.; Sharma, V.; Kansal, L. The protective effect of Rubia cordifolia against lead nitrate-induced immune response impairment and kidney oxidative damage. Indian J. Pharmacol. 2011, 43, 441-444. [CrossRef] [PubMed] 
14. Lopez-Exposito, I.; Castillo, A.; Yang, N.; Liang, B.H.; Li, X.M. Chinese herbal extracts of Rubia cordifolia and Dianthus superbus suppress IgE production and prevent peanut-induced anaphylaxis. Chin. Med. 2011. [CrossRef] [PubMed]

15. Shilpa, P.N.; Venkatabalasubramanian, S.; Devaraj, S.N. Induction of apoptosis by methanolic extract of Rubia Cordifolia Linn in HEp-2 cell line is mediated by reactive oxygen species. Asian Pac. J. Cancer Prev. 2012, 13, 2753-2758. [CrossRef] [PubMed]

16. Shilpa, P.N.; Venkatabalasubramanian, S.; Devaraj, S.N. Ameliorative effect of methanol extract of Rubia cordifolia in N-nitrosodiethylamine-induced hepatocellular carcinoma. Pharm. Biol. 2012, 50, 376-383. [CrossRef] [PubMed]

17. Zhu, Z.G.; Jin, H.; Yu, P.J.; Tian, Y.X.; Zhang, J.J.; Wu, S.G. Mollugin inhibits the inflammatory response in lipopolysaccharide-stimulated RAW264.7 macrophages by blocking the Janus kinase-signal transducers and activators of transcription signaling pathway. Biol. Pharm. Bull. 2013, 36, 399-406. [CrossRef] [PubMed]

18. Gupta, D.; Kumari, S.; Gulrajani, M. Dyeing studies with hydroxyanthraquinones extracted from Indian madder. Part 1: Dyeing of nylon with purpurin. Color. Technol. 2001, 117, 328-332. [CrossRef]

19. Gupta, D.; Kumari, S.; Gulrajani, M. Dyeing studies with hydroxyanthraquinones extracted from Indian madder. Part 2: Dyeing of nylon and polyester with nordamncanthal. Color. Technol. 2001, 117, 333-336. [CrossRef]

20. Vankar, P.S.; Shanker, R.; Mahanta, D.; Tiwari, S.C. Ecofriendly sonicator dyeing of cotton with Rubia cordifolia Linn using biomordant. Dyes Pigment 2008, 76, 207-212. [CrossRef]

21. Yusuf, M.; Shahid, M.; Khan, S.A.; Khan, M.I.; Shahid-Ul-Islam; Mohammad, F.; Khan, M.A. Eco-dyeing of wool using aqueous extract of the roots of Indian Madder (Rubia cordifolia) as natural dye. J. Nat. Fibers 2013, 10, 14-28. [CrossRef]

22. Namsa, N.D.; Mandal, M.; Tangjang, S.; Mandal, S.C. Ethnobotany of the Monpa ethnic group at Arunachal Pradesh, India. J. Ethnobiol. Ethnomed. 2011. [CrossRef] [PubMed]

23. Xun, L. Quality Control Study of Rubia cordifolia L. and the Fakes. Master's Thesis, Beijing University of Chinese Medicine, Beijing, China, June 2014.

24. State Administration of Traditional Chinese Medicine. Chinese Materia Medica, 1st ed.Shanghai Scientific \& Technical Publishers: Shanghai, China, 1998; Volume 18, pp. 470-475.

25. Chen, S.B.; Feng, R.Z.; Chen, B.Z.; Jing, L.H.; Dong, X.; Gu, Z.P.; Wang, S.B. A study on the medicinal plants of genus Rubia. I. Botanical origins and resource of Chinese traditional drug “Qiancao" (Madder). Nat. Prod. Res. Dev. 1991, 3, 7-15.

26. Arisawa, M.; Ueno, H.; Nimura, M.; Hayashi, T.; Morita, N. Rubiatriol, a new triterpenoid from the Chinese drug, Qian-Cao-Gen, Rubia cordifolia. J. Nat. Prod. 1986, 74, 2069-2080. [CrossRef]

27. Gandhi, M.R.; Reegan, A.D.; Ganesan, P.; Sivasankaran, K.; Paulraj, M.G.; Balakrishna, K.; Ignacimuthu, S.; Al-Dhabi, N.A. Larvicidal and pupicidal activities of alizarin isolated from roots of Rubia cordifolia against Culex quinquefasciatus Say and Aedes aegypti (L.) (Diptera: Culicidae). Neotrop. Entomol. 2016, 45, 441-448. [CrossRef] [PubMed]

28. Murti, V.V.S.; Seshadri, T.R.; Sivakuma, S. Anthraquinones of Rubia cordifolia. Phytochemistry 1972, 11, 1524. [CrossRef]

29. Son, J.K.; Jung, S.J.; Jung, J.H.; Fang, Z.; Lee, C.S.; Seo, C.S.; Moon, D.C.; Min, B.S.; Kim, M.R.; Woo, M.H. Anticancer Constituents from the Roots of Rubia cordifolia L. Chem. Pharm. Bull. 2008, 56, 213-216. [CrossRef] [PubMed]

30. Wang, S.X.; Hua, H.M.; Wu, L.J.; Li, X.; Zhu, T.R. Study on anthraquinones from the roots of Rubia cordifolia L. Acta Pharm. Sin. 1992, 27, 743-747.

31. Mischenko, N.P.; Fedoreyev, S.A.; Glazunov, V.P.; Chernoded, G.K.; Bulgakov, V.P.; Zhuravlev, Y.N. Anthraquinone production by callus cultures of Rubia cordifolia. Fitoterapia 1999, 70, 552-557. [CrossRef]

32. Tessier, A.M.; Delaveau, P.; Champion, B. New anthraquinones in Rubia cordifolia roots. Planta Med. 1981, 41, 337-343. [CrossRef] [PubMed]

33. Jiang, Z.; Han, D.Z.; Jin, G.Z. Study on the chemical constituents and anticancer activity from the roots of Rubia cordifolia L. Chin. Hosp. Pharm. J. 2012, 32, 1126-1128.

34. Li, X.; Liu, Z.; Chen, Y.; Wang, L.J.; Zheng, Y.N.; Sun, G.Z.; Ruan, C.C. Rubiacordone A: A new anthraquinone glycoside from the roots of Rubia cordifolia. Molecules 2009, 14, 566-572. [CrossRef] [PubMed] 
35. Dosseh, C.; Tessier, A.M.; Delaveau, P. New quinones in Rubia cordifolia L. Roots, III. Planta Med. 1981, 43, 360-363. [CrossRef] [PubMed]

36. Koyama, J.; Ogura, T.; Tagahara, K.; Konoshima, T.; Kozuka, M. Two naphthoquinones from Rubia cordifolia. Phytochemistry 1992, 31, 2907-2908. [CrossRef]

37. Liu, R.; Lu, Y.B.; Wu, T.X.; Pan, Y.J. Simultaneous isolation and purification of mollugin and two anthraquinones from Rubia cordifolia by HSCCC. Chromatographia 2008, 68, 95-99. [CrossRef]

38. Lu, Y.B.; Hu, R.L.; Dai, Z.Y.; Pan, Y.J. Preparative separation of anti-oxidative constituents from Rubia cordifolia by column-switching counter-current chromatography. J. Sep. Sci. 2010, 33, 2200-2205. [CrossRef] [PubMed]

39. Lin, S.Q.; Gao, J.F.; Wu, L.; Yuan, X. Study of anthraquinone components from rhizome of Rubia cordifolia by preparative chromatogram of medium and low press. Guihaia 2011, 31, 857-860.

40. Dosseh, C.; Tessier, A.M.; Delaveau, P. Rubia cordifolia roots. II: New quinones. Planta Med. 1981, 43, $141-147$. [CrossRef] [PubMed]

41. Wang, J.J. Study on the Chemical and Biological Activity of Rubia cordifolia L. Collected from Henan Province. Master's Thesis, Henan University, Kaifeng, China, June 2014.

42. Abdullah, S.T.; Ali, A.; Hamid, H.; Ali, M.; Ansari, S.H.; Alam, M.S. Two new anthraquinones from the roots of Rubia cordifolia Linn. Pharmazie 2003, 58, 216-217. [PubMed]

43. Son, J.K.; Jung, J.H.; Lee, C.S.; Moon, D.C.; Choi, S.W.; Min, B.S.; Woo, M.H. DNA Topoisomerases I and II inhibition and cytotoxicity of constituents from the roots of Rubia cordifolia. Bull. Korean Chem. Soc. 2006, 27, 1231-1234. [CrossRef]

44. Gupta, P.P.; Srimal, R.C.; Verma, N.; Tandon, J.S. Biological activity of Rubia cordifolia and isolation of an active principle. Pharm. Biol. 1999, 37, 46-49. [CrossRef]

45. Ho, L.K.; Don, M.J.; Chen, H.C.; Yeh, S.F.; Chen, J.M. Inhibition of hepatitis B surface antigen secretion on human hepatoma cells. Components from Rubia cordifolia. J. Nat. Prod. 1996, 59, 330-333. [CrossRef] [PubMed]

46. Wu, L.J.; Wang, S.X.; Hua, H.M.; Li, X.; Zhu, T.R.; Miyase, T.; Ueno, A. 6-Methoxygeniposidic acid, an iridoid glycoside from Rubia cordifolia. Phytochemistry 1991, 30, 1710-1711. [CrossRef]

47. Jun, D.Y.; Han, C.R.; Lee, J.Y.; Park, W.; Choi, M.S.; Woo, M.H.; Kim, Y.H. Anti-adipogenic activity of 2-carbomethoxy-2,3-epoxy-3-prenyl-1,4-naphthoquinone from Rubia cordifolia L. J. Med. Food 2011, 14, 454-461. [CrossRef] [PubMed]

48. Itokawa, H.; Takeya, K.; Mori, N.; Sonobe, T.; Mihashi, S.; Hamanaka, T. Studies on antitumor cyctic hexapeptides RA obtained from Rubiae Radix, Rubiaceae.VI. Minor antitumor constituents. Chem. Pharm. Bull. 1986, 34, 3762-3768. [CrossRef] [PubMed]

49. Itokawa, H.; Morita, H.; Takeya, K.; Tomioka, N.; Itai, A.; Iitaka, Y. New antitumor bicyclic hexapeptides, RA-VI and RA-VIII from Rubia cordifolia-conformation-activity relationship II. Tetrahedron 1991, 47, 7007-7020. [CrossRef]

50. Itokawa, H.; Yamamiya, T.; Morita, H.; Takeya, K. New antitumour bicyclic hexapeptides, RA-IX and -X from Rubia cordifolia. Part 3. Conformation-antitumour activity relationship. J. Chem. Soc. Perkin Trans. 1992, 14, 455-459. [CrossRef]

51. Morita, H.; Yamamiya, T.; Takeya, K.; Itokawa, H. New antitumor bicyclic hexapeptides, RA-XI, RA-XII, RA-XIII and RA-XIV from Rubia cordifolia. Chem. Pharm. Bull. 1992, 40, 1352-1354. [CrossRef] [PubMed]

52. Takeya, K.; Yamamiya, T.; Morita, H.; Itokawa, H. Two antitumour bicyclic hexapeptides from Rubia cordifolia. Phytochemistry 1993, 33, 613-615. [CrossRef]

53. Hitotsuyanagi, Y.; Ishikawa, H.; Hasuda, T.; Takeya, K. Isolation, structural elucidation, and synthesis of RA-XVII, a novel bicyclic hexapeptide from Rubia cordifolia, and the effect of side chain at residue 1 upon the conformation and cytotoxic activity. Tetrahedron Lett. 2004, 45, 935-938. [CrossRef]

54. Lee, J.E.; Hitotsuyanagi, Y.; Kim, I.H.; Hasuda, T.; Takeya, K. A novel bicyclic hexapeptide, RA-XVIII, from Rubia cordifolia: Structure, semi-synthesis, and cytotoxicity. Bioorg. Med. Chem. Lett. 2008, 18, 808-811. [CrossRef] [PubMed]

55. Lee, J.E.; Hitotsuyanagi, Y.; Takeya, K. Structures of cytotoxic bicyclic hexapeptides, RA-XIX, -XX, -XXI, and -XXII, from Rubia cordifolia L. Tetrahedron 2008, 64, 4117-4125. [CrossRef]

56. Lee, J.E.; Hitotsuyanagi, Y.; Fukaya, H.; Kondo, K.; Takeya, K. New cytotoxic bicyclic hexapeptides, RA-XXIII and XXIV, from Rubia cordifolia L. Chem. Pharm. Bull. 2008, 56, 730-733. [CrossRef] [PubMed] 
57. Itokawa, H.; Morita, H.; Takeya, K.; Tomioka, N.; Itai, A. RAI-III and VI, Conformational isomers of antitumor cyclic hexapeptides, RA-III and VI from Rubia cordifolia. Chem. Lett. 1991, 12, 2217-2220. [CrossRef]

58. Hitotsuyanagi, Y.; Aihara, T.; Takeya, K. RA-dimer A, a novel dimeric antitumor bicyclic hexapeptide from Rubia cordifolia L. Tetrahedron Lett. 2000, 41, 6127-6130. [CrossRef]

59. Itokawa, H.; Qiao, Y.F.; Takeya, K. New arborane type triterpenoids from Rubia cordifolia var Pratensis and Rubia oncotricha. Chem. Pharm. Bull. 1990, 38, 1435-1437. [CrossRef]

60. Itokawa, H.; Qiao, Y.F.; Takeya, K.; Iitaka, Y. New triterpenoids from Rubiacordifolia var Pratensis (Rubiaceae). Chem. Pharm. Bull. 1989, 37, 1670-1672. [CrossRef]

61. Ibraheim, Z.Z.; Gouda, Y.G. Minor constituents from Rubia cordifolia L. root. Bull. Pharm. Sci. 2010, 33, 225-233.

62. Wang, S.X.; Hua, H.M.; Wu, L.J.; Li, X.; Zhu, T.R.; Miyase, T.; Ueno, A. Structure identification of a new iridoid glycoside from Rubia cordifolia. J. Shenyang Coll. Pharm. 1991, 46, 58.

63. Hassanean, H.A.; Ibraheim, Z.Z.; Takeya, K.; Itorawa, H. Further quinoidal derivatives from Rubia cordifolia L. Pharmazie 2000, 55, 317-319. [PubMed]

64. Chang, L.C.; Chavez, D.; Gills, J.J.; Fong, H.H.S.; Pezzuto, J.M.; Kinghorn, A.D. Rubiasins A-C, new anthracene derivatives from the roots and stems of Rubia cordifolia. Tetrahedron Lett. 2000, 41, 7157-7162. [CrossRef]

65. Huang, R.Q.; Wang, Z.H.; Wang, H.X. Studies on the constituents of polysaccharides RPS-1, RPS-2 and RPS-3 from R. cordifolia. Chin. Tradit. Patent Med. 1996, 19, 25-26.

66. Wang, H.X.; Wang, B. Isolation, purification and structural analysis of polysaccharide QA2 from Indian Madder (Rubia cordifolia). Chin. Tradit Herb. Drugs 1998, 29, 219-221.

67. Park, H.; Shim, J.S.; Kim, B.S.; Jung, H.J.; Huh, T.L.; Kwon, H.J. Purpurin inhibits adipocyte-derived leucineaminopeptidase and angiogenesis in a zebrafish model. Biochem. Biophys. Res. Commun. 2014, 450, 561-567. [CrossRef] [PubMed]

68. Jeremic, S.R.; Sehovic, S.F.; Manojlovic, N.T.; Markovic, Z.S. Antioxidant and free radical scavenging activity of purpurin. Monatshefte Chem. 2012, 143, 427-435. [CrossRef]

69. Zengin, G.; Degirmenci, N.S.; Alpsoy, L.; Aktumsek, A. Evaluation of antioxidant, enzyme inhibition, and cytotoxic activity of three anthraquinones (alizarin, purpurin, and quinizarin). Hum. Exp. Toxicol. 2016, 35, 544-553. [CrossRef] [PubMed]

70. Do, M.T.; Hwang, Y.P.; Kim, H.G.; Na, M.; Jeong, H.G. Mollugin inhibits proliferation and induces apoptosis by suppressing fatty acid synthase in HER2-overexpressing cancer cells. J. Cell. Physiol. 2013, 228, 1087-1097. [CrossRef] [PubMed]

71. Zhang, L.; Wang, H.D.; Zhu, J.H.; Xu, J.G.; Ding, K. Mollugin induces tumor cell apoptosis and autophagy via the PI3K/AKT/mTOR/p70S6K and ERK signaling pathways. Biochem. Biophys. Res. Commun. 2014, 450, 247-254. [CrossRef] [PubMed]

72. Jeong, G.S.; Lee, D.S.; Kim, D.C.; Jahng, Y.; Son, J.K.; Lee, S.H.; Kim, Y.C. Neuroprotective and anti-inflammatory effects of mollugin via up-regulation of heme oxygenase- 1 in mouse hippocampal and microglial cells. Eur. J. Pharmacol. 2011, 654, 226-234. [CrossRef] [PubMed]

73. Kim, K.J.; Lee, J.S.; Kwak, M.K.; Choi, H.G.; Yong, C.S.; Kim, J.A.; Lee, Y.R.; Lyoo, W.S.; Park, Y.J. Anti-inflammatory action of mollugin and its synthetic derivatives in HT-29 human colonic epithelial cells is mediated through inhibition of NF-kappa B activation. Eur. J. Pharmacol. 2009, 622, 52-57. [CrossRef] [PubMed]

74. Tripathi, Y.B.; Singh, A.V. Role of Rubia cordifolia Linn in radiation protection. Indian J. Exp. Biol. 2007, 45, 620-625. [PubMed]

75. Baskar, R.; Bhakshu, L.M.; Bharathi, G.V.; Reddy, S.S.; Karuna, R.; Reddy, G.K.; Saralakumari, D. Antihyperglycemic activity of aqueous root extract of Rubia cordifolia in streptozotocin-induced diabetic rats. Pharm. Biol. 2006, 44, 475-479. [CrossRef]

76. Rao, G.M.M.; Rao, C.V.; Pushpangadan, P.; Shirwaikar, A. Hepatoprotective effects of rubiadin, a major constituent of Rubia cordifolia Linn. J. Ethnopharmacol. 2006, 103, 484-490. [CrossRef] [PubMed]

77. Tripathi, Y.B.; Shukla, S.D. Rubia cordifolia extract inhibits cell proliferation in A-431 cells. Phytother. Res. 1998, 12, 454-456. [CrossRef]

78. Tripathi, Y.B.; Pandey, S.; Shukla, S.D. Anti-platelet activating factor property of Rubia cordifolia Linn. Indian J. Exp. Biol. 1993, 31, 533-535. [PubMed] 
79. Deshkar, N.; Tilloo, S.; Pande, V. A comeprehensive review of Rubia cordifolia Linn. Pharmacogn. Rev. 2008, 2, 124-135.

80. Patil, R.; Mohan, M.; Kasture, V.; Kasture, S. Rubia cordifolia: A review. Orient. Pharm. Exp. Med. 2009, 9, 1-13. [CrossRef]

81. Shan, M.Q.; Chen, X.; Li, J.; Yu, B.; Ding, A.W. Comparative study on effects of Rubiae Radix et Rhizoma and carbonized Rubiae Radix et Rhizoma on acute blood stasis rat model. China J. Chin. Mater. Med. 2014, 39, 493-497.

82. Geng, Q.B.; Huang, H.L.; Weng, Z.F.; Chen, P.; Zhang, W.Z.; Cai, Y.C. Effects of Rubia cordifolia and charred R. Cordifolia on coagulation-fibrinolytic system of normal rats. Chin. J. Exp. Tradit. Med. Formulae 2013, 19, 279-282.

83. Nishimura, N.; Takai, M.; Yamamoto, E.; Hasumi, K. Purpurin as a specific inhibitor of spermidine-induced autoactivation of the protease plasma hyaluronan-binding protein. Biol. Pharm. Bull. 2010, 33, 1430-1433. [CrossRef] [PubMed]

84. Kaur, P.; Chandel, M.; Kumar, S.; Kumar, N.; Singh, B.; Kaur, S. Modulatory role of alizarin from Rubia cordifolia L. against genotoxicity of mutagens. Food Chem. Toxicol. 2010, 48, 320-325. [CrossRef] [PubMed]

85. Lee, Y.M.; Auh, Q.S.; Lee, D.W.; Kim, J.Y.; Jung, H.J.; Lee, S.H.; Kim, E.C. Involvement of Nrf2-mediated upregulation of hemeoxygenase-1 in mollugin-induced growth inhibition and apoptosis in human oral cancer cells. Biomed. Res. Int. 2013. [CrossRef] [PubMed]

86. Ghosh, S.; Sarma, M.D.; Patra, A.; Hazra, B. Anti-inflammatory and anticancer compounds isolated from Ventilago madraspatana Gaertn, Rubia cordifolia Linn and Lantana camara Linn. J. Pharm. Pharmacol. 2010, 62, 1158-1166. [CrossRef] [PubMed]

87. Pawar, A.T.; Anap, R.M.; Ghodasara, J.V.; Kuchekar, B.S. Protective effect of hydroalcoholic root extract of Rubia cordifolia in indomethacin-induced enterocolitis in rats. Indian J. Pharm. Sci. 2011, 73, 250-253. [CrossRef] [PubMed]

88. Patil, R.A.; Kasture, S.B. Protective effect of Rubia cordifolia on reserpine-induced orofacial dyskinesia. Nat. Prod. Res. 2012, 26, 2159-2161. [PubMed]

89. Cai, Y.; Sun, M.; Xing, J.; Corke, H. Antioxidant phenolic constituents in roots of Rheum officinale and Rubia cordifolia: Structure-radical scavenging activity relationships. J. Agric. Food Chem. 2004, 52, 7884-7890. [CrossRef] [PubMed]

90. Divakar, K.; Pawar, A.T.; Chandrasekhar, S.B.; Dighe, S.B.; Divakar, G. Protective effect of the hydro-alcoholic extract of Rubia cordifolia roots against ethylene glycol induced urolithiasis in rats. Food Chem. Toxicol. 2010, 48, 1013-1018. [CrossRef] [PubMed]

91. Lin, Z.X.; Jiao, B.W.; Che, C.T.; Zuo, Z.; Mok, C.F.; Zhao, M.; Ho, W.K.K.; Tse, W.P.; Lam, K.Y.; Fan, R.Q.; et al. Ethyl acetate fraction of the root of Rubia cordifolia L. inhibits keratinocyte proliferation in vitro and promotes keratinocyte differentiation in vivo: Potential application for psoriasis treatment. Phytother. Res. 2010, 24, 1056-1064. [PubMed]

92. Joy, J.; Nair, C.K. Amelioration of cisplatin induced nephrotoxicity in Swiss albino mice by Rubia cordifolia extract. J. Cancer Res. Ther. 2008, 4, 111-115. [PubMed]

93. Parial, S.D.; Sharma, D.K.; Mistry, S.; Middha, A.; Agarwal, K. Estrogenic and progestational activity of roots of Rubia cordifolia. Asian J. Chem. 2007, 19, 2861-2865.

94. Baek, J.M.; Kim, J.Y.; Jung, Y.; Moon, S.H.; Choi, M.K.; Kim, S.H.; Lee, M.S.; Kim, I.; Oh, J. Mollugin from Rubia cordifolia suppresses receptor activator of nuclear factor-kappa B ligand-induced osteoclastogenesis and bone resorbing activity in vitro and prevents lipopolysaccharide-induced bone loss in vivo. Phytomedicine 2015, 22, 27-35. [CrossRef] [PubMed]

95. Sabde, S.; Bodiwala, H.S.; Karmase, A.; Deshpande, P.J.; Kaur, A.; Ahmed, N.; Chauthe, S.K.; Brahmbhatt, K.G.; Phadke, R.U.; Mitra, D.; et al. Anti-HIV activity of Indian medicinal plants. J. Nat. Med. 2011, 65, 662-669. [CrossRef] [PubMed]

96. Inoue, K.; Yoshida, M.; Takahashi, M.; Fujimoto, H.; Ohnishi, K.; Nakashima, K.; Shibutani, M.; Hirose, M.; Nishikawa, A. Possible contribution of rubiadin, a metabolite of madder color, to renal carcinogenesis in rats. Food Chem. Toxicol. 2009, 47, 752-759. [CrossRef] [PubMed] 
97. Inoue, K.; Yoshida, M.; Takahashi, M.; Fujimoto, H.; Shibutani, M.; Hirose, M.; Nishikawa, A. Carcinogenic potential of alizarin and rubiadin, components of madder color, in a rat medium-term multi-organ bioassay. Cancer Sci. 2009, 100, 2261-2267. [CrossRef] [PubMed]

98. Li, C.C.; Li, J.S.; Huang, G.X.; Yan, L.J.; Wang, Q.Q. Study on potential toxicity of madder pigment. Guangdong Agric. Sci. 2013, 40, 35-36, 39.

99. Wu, D.Z.; Liu, Q.D.; Zhang, X.G.; Liu, D.P.; Liu, L.L. The pharmacokinetics and leukocytotic effect of 1,4-naphthodiol-2,3-dicarboxylic acid diethyl ester (NDDE). Bull. Acad. Mil. Med. Sci. 1984, 32, 437-442.

100. Zhang, Z.H. Clinical observation of diethylester Rubia cordifolia L. promoting the increase of leucocyte count in leucopenia. Chin. J. Integr. Tradit. West. Med. 1983, 3, 98-99.

101. Xie, Y.Q. Rubidatum. Pharm. Ind. 1987, 18, 92-93.

102. Haruna, K.; Kanezaki, H.; Tanabe, K.; Dai, W.M.; Nishimoto, S. Effects of structural modification on the DNA binding properties and photo-induced cleavagere activity of propargylic sulfones conjugated with an anthraquinone structure. Bioorg. Med. Chem. 2006, 14, 4427-4432. [CrossRef] [PubMed]

103. Rajendran, A.; Gandhidasan, R.; Murugesan, R. Photosensitisation and photoinduced DNA cleavage by four naturally occurring anthraquinones. J. Photochem. Photobiol. A 2004, 168, 67-73. [CrossRef]

(C) 2016 by the authors; licensee MDPI, Basel, Switzerland. This article is an open access article distributed under the terms and conditions of the Creative Commons Attribution (CC-BY) license (http://creativecommons.org/licenses/by/4.0/). 